Comportamento assintótico para soluções de certas equações diferenciais funcionais periódicas

\author{
Juliano Ribeiro de Oliveira
}



SERVIÇO DE PÓS-GRADUAÇÃO DO ICMC-USP

Data de Depósito: 27 de Fevereiro de 2008

Assinatura:

\title{
Comportamento assintótico para soluções de certas equações diferenciais funcionais periódicas
}

\author{
Juliano Ribeiro de Oliveira
}

Orientador: Prof. Dr. Miguel Vinícius Santini Frasson

Dissertação apresentada ao Instituto de Ciências Matemáticas e de Computação - ICMC/USP, como parte dos requisitos para obtenção do título de Mestre em Matemática.

USP - São Carlos

Fevereiro/2008 

Aos meu pais João e Ana. 



\section{Agradecimentos}

Agradeço à toda minha família, pelo apoio e união. De maneira especial aos meus pais João e Ana pela grande educação que me deram. À minha querida tia Gercina que sempre ajudou na minha criação. Ao meu irmão pelo companheirismo e amizade. À minha namorada Tamiris, que teve que suportar a distância que nos separou durante esses anos, e à sua família que sempre me apoiou.

Agradeço a todos os meus amigos, em especial, ao Pimenta, com quem estudo desde 1999, quando fizemos o SENAI, depois a faculdade e agora o mestrado. Ao cabeçudo do Alex, companheiro de cidade natal, futebol, brincadeiras e trapalhadas. Ao Cláudio, com quem dividi a mesma república durante este tempo. Ao Hartmamm e ao Ubarana, companheiros de sala e de aventuras na bolsa de valores. Ao sempre irreverente Juliano Oler. Ao Dusse, também companheiro de rep. À minha turma (Giu, Duzinho, Thiago, Leitão, Lucas, Thaís, Wescley, Yuri). Ao pessoal do futebol (Prof. Marcelo Saia, Aldício, Chalapa, Sandrão da cantina, Walter, João Paulo, Cássio, Lacassa, Fernando, Pedrão, Kengi, Homero, Jean).

Agradeço também a todos os professores, funcionários e amigos de todas escolas nas quais já estudei, ao pessoal da E.E. Prof. Ivo Liboni, onde estudei 11 anos, da escola profissionalizante SENAI, da UNESP de Presidente Prudente, e agora do ICMC-USP. Gostaria de agradecer à Capes pelo apoio financeiro. Por fim, agradeço de maneira especial ao meu orientador Miguel Vinícius Santini Frasson, que me ajudou muito nesta caminhada, teve paciência e que até dispôs de seu tempo livre para me ajudar. 



\section{Resumo}

Estamos interessados em estudar o comportamento assintótico das soluções de uma classe de Equações Diferenciais Funcionais (EDF) lineares e autônomas do tipo neutro, onde os coeficientes, na parte não neutra, são funções periódicas de período comum $\omega$ e os retardamentos são múltiplos de $\omega$. Para isto, utilizamo-nos da teoria espectral de operadores aplicada ao chamado operador monodrômico $\Pi: \mathcal{C} \rightarrow \mathcal{C}$, cuja ação é evoluir um dado estado um passo de tamanho $\omega$. Calculamos o resolvente deste operador, donde inferimos todas as propriedades espectrais que nos permitem determinar o comportamento assintótico das soluções. Mostramos a importância de se determinar autovalores dominantes para a obtenção das estimativas, e mostramos resultados neste sentido. Estudamos em detalhe três exemplos que ilustram a teoria e demonstram sua aplicabilidade.

Palavras-chave: Equações diferenciais funcionais, equações periódicas, comportamento assintótico, teoria espectral, dominância de autovalores. 



\section{Abstract}

We are interested in the study of the asymptotic behavior of the solutions of a class of linear autonomous Functional Differential Equations (FDE) of neutral type, where the coefficients of the non neutral part are periodic functions with common period $\omega$ and the time delays are multiples of $\omega$. We employ the spectral theory for linear operators applied to the so called monodromic operator $\Pi: \mathcal{C} \rightarrow \mathcal{C}$, whose action is to evolve a given state one step of size $\omega$. We compute the resolvent of this operator, from where we infer the spectral properties that allows us to determine the asymptotic behavior of the solutions. We show the importance to determine whether an eigenvalue is dominant, in order to obtain the estimates for the correspondet solution, and we show results in this direction. Finally we study in detail three examples that illustrate the theory and demonstrate its applicability.

Keywords: Functional differential equations, periodic equations, asymptotic behavior, spectral theory, dominance of eigenvalues. 


\section{Sumário}

$\begin{array}{ll}\text { Apresentação } & 11\end{array}$

1 Teoria Básica de Equações Diferenciais Funcionais 13

1.1 Introdução . . . . . . . . . . . . . . . . . . . 13

1.2 Teoria qualitativa . . . . . . . . . . . . . 16

1.3 Sistemas lineares gerais . . . . . . . . . . . . . . . 17

1.4 Operador solução para sistemas lineares . . . . . . . . . 18

2 Sistemas Periódicos $\quad 21$

2.1 Sistemas periódicos com retardamento . . . . . . . . . 21

2.2 Sistemas periódicos neutros . . . . . . . . . . . 28

3 Uma Classe de Sistemas Periódicos $\quad 31$

3.1 Teoria espectral . . . . . . . . . . . . . . . . 31

3.2 O operador monodrômico . . . . . . . . . . . . 34

3.3 Comportamento assintótico . . . . . . . . . . 38

4 Algumas Aplicações $\quad 43$

4.1 Um exemplo estudado . . . . . . . . . . . . . . . . . 43

4.2 Exemplo sem autoespaços de dimensão finita . . . . . . . . . . 47

4.3 Certas equaçõos periódicas simples . . . . . . . . . . . . 48

A Dominância de Raízes de Equações Características $5 \mathbf{5 1}$

A.1 Uma condição para a dominância de raízes . . . . . . . . . . 52

A.2 Uma equação simples . . . . . . . . . . . . . . . . . . . 55

$\begin{array}{lr}\text { Referências } & 59\end{array}$

$\begin{array}{lc}\text { İndice Remissivo } & 61\end{array}$ 


\section{Apresentação}

Estamos interessados no estudo das soluções das Equações Diferenciais Funcionais (EDF) do tipo neutro, na forma

$$
\frac{d}{d t}\left(x(t)+\sum_{i=1}^{k} c_{i} x(t-i \omega)\right)=a(t) x(t)+\sum_{i=1}^{k} b_{i}(t) x(t-i \omega),
$$

onde as funções $a(t)$ e $b_{i}(t), i=1,2, \ldots, k$, são contínuas e $\omega$-periódicas, $c_{i}$, $i=0,1, \ldots, k$, (assumimos $c_{0}=1$ ) são números reais, não necessariamente não-nulos. Notamos que os retardamentos são múltiplos do período $\omega$.

Sob a restrição de que as constantes $c_{i}, i=1, \ldots, k$, são nulas, o comportamento assintótico das soluções de (1) foi estudado por Hale \& Verduyn Lunel [6] e Frasson \& Verduyn Lunel [3]. Baseamo-nos principalmente nas idéias usadas em [3], implementando as adaptações necessárias para incorporar os retardamentos na parte neutra.

Para esta classe de equações, recentemente Philos \& Purnaras [8] publicaram resultados interessantes. Este trabalho inspirou a forma da equação (1) estudada neste trabalho.

O método que empregamos é o estudo do comportamento assintótico baseado na análise das propriedades espectrais do operador monodrômico, que evolui um determinado estado, partindo de $t=0$ até $t=\omega$. Mostramos que o comportamento assintótico é obtido desde que se conheça que um determinado autovalor $\mu_{0}$ do operador monodrômico é dominante, isto é, existe $\epsilon>0$ tal que todo outro autovalor $\mu$ satisfaz $|\mu|<\left|\mu_{0}\right|-\epsilon$. Também, $\mu_{0}$ não pode ser autovalor de um operador solução da equação diferença associado a (1). No Apêndice A, apresentamos resultados que implicam a dominância de um determinado zero de funções que tipicamente aparecem como equações características de equações diferenciais funcionais.

Em [8], utilizando-se de outros meios distintos dos nossos, seus autores obtêm o comportamento assintótico das soluções que podem ser obtidos com a 
combinação dos resultados aqui apresentados no Capítulo 3 e no Apêndice A. Nossos resultados são mais gerais, visto que pode-se obter a dominância por outros meios. Na Seção 4.1, por exemplo, estudamos uma EDF em que não seria possível derivar o comportamento assintótico das soluções utilizando-se apenas os resultados contidos em [8], pois não há zero que satisfaça a hipótese $\left(\mathrm{P}\left(\lambda_{0}\right)\right)$ do Teorema 1 de [8]. No entanto, fomos capazes de encontrar o autovalor dominante e derivar o comportamento assintótico das soluções.

Esta dissertação está organizada da seguinte maneira. No Capítulo 1, apresentamos brevemente a teoria básica das Equações Diferenciais Funcionais, com atenção especial às equações lineares. No Capítulo 2, apresentamos a teoria das EDF lineares periódicas retardadas com forma bastante geral, como encontramos no livro de Hale \& Verduyn Lunel [6], assim como generalizações que funcionam também para equações lineares periódicas do tipo neutro. No Capítulo 3, generalizamos alguns resultados de Frasson \& Verduyn Lunel [3], calculando o operador monodrômico associado à equação (1) e seu resolvente. Daí, utilizando da teoria de operadores lineares, obtivemos as estimativas necessárias. No Capítulo 4, estudamos em detalhe três exemplos, que ilustram a aplicabilidade dos resultados. No primeiro exemplo, tivemos que provar a dominância de um determinado autovalor para derivarmos o comportamento assintótico das soluções. No segundo exemplo, mostramos uma classe de equações onde todos os autovalores do operador monodrômico são autovalores também dos operadores referentes à equação neutra associada. Finalmente, estudamos uma classe de equações retardadas periódicas com um retardamento, obtendo resultados que generalizam outros resultados na literatura. Apresentamos ainda um apêndice onde são mostrados resultados auxiliares para a obtenção da dominância de um zero de uma função. Um destes resultados foi usado no terceiro exemplo do Capítulo 4. 


\section{Capítulo 1}

\section{Teoria Básica de Equações Diferenciais Funcionais}

Neste capítulo, introduzimos a teoria básica das Equações Diferenciais Funcionais (EDF), com uma discussão de suas principais propriedades. Apresentaremos alguns resultados sobre existência, unicidade, continuação e dependência contínua de soluções das equações diferenciais funcionais. Quando há existência e unicidade de soluções de uma EDF, fica motivada a definição do chamado operador solução, que é um semigrupo fortemente contínuo. Estudaremos algumas propriedades destes operadores.

\subsection{Introdução}

Em muitas aplicações, assume-se que o sistema considerado é governado pelo princípio da causalidade, isto é, o estado futuro do sistema é determinado somente pelo presente, sendo independente do passado. Se assumimos que o sistema é governado por uma equação envolvendo o estado e a taxa de variação deste estado, então geralmente consideramos equações diferenciais ordinárias ou parciais. Entretanto, num exame mais detalhado do sistema, torna-se aparente que o princípio da causalidade é sempre uma primeira aproximação da situação real e que um modelo mais realista incluiria a dependência em algum estado passado do sistema. Também, pela natureza de alguns problemas, não faz sentido que não se considere o passado. Isto é conhecido há muito tempo mas a teoria para tais sistemas tem sido bastante desenvolvida apenas recentemente. Este é o ramo das Equações Diferenciais Funcionais. Para uma exposição introdutória, veja $[6,1]$ 
Um sistema dos mais simples modelado nestes moldes pode ser descrito pela equação diferencial

$$
\dot{x}(t)=A x(t)+B x(t-r),
$$

onde $A, B$ e $r>0$ são constantes e $x$ é escalar. A primeira questão que nos vem é qual é o problema de valor inicial para a equação (1.1), isto é, qual é a quantidade mínima de informação que devemos dar para que a equação (1.1) defina $x(t)$ para algum $t \geqslant 0$ ? Com um pouco de reflexão chega-se à conclusão de que uma função deve ser especificada para todo intervalo $[-r, 0]$. Conhecida a solução em um intervalo $[t-r, t]$, podemos "resolver" $\dot{x}(t)$, podendo assim "integrar" a equação. Então é natural tomar o estado em determinado instante $t$ como sendo a história da solução em $[t-r, t]$. Isto motiva a olhar a equação (1.1) como um sistema dinâmico em um espaço de funções definidas em $[-r, 0]$. A seguir, apresentaremos uma pequena introdução à teoria básica destas equações.

Seja $\mathcal{C} \stackrel{\text { def }}{=} \mathcal{C}\left([-r, 0], \mathbb{R}^{n}\right)$ o espaço de Banach das funções contínuas de $[-r, 0](r>0)$ a valores em $\mathbb{R}^{n}$, munido da norma do supremo. Se $\sigma \in \mathbb{R}$, $A>0$ e $x \in \mathcal{C}\left([\sigma-r, \sigma+A], \mathbb{R}^{n}\right)$, para qualquer $t \in[\sigma, \sigma+A]$, definimos $x_{t} \in \mathcal{C}$ por $x_{t}(\theta)=x(t+\theta)$, para $\theta \in[-r, 0]$. Do Teorema da Representação de Riesz (veja por exemplo Royden [9] ou Rudin [10]), toda aplicação linear contínua $L: \mathcal{C} \rightarrow \mathbb{R}^{n}$ pode ser representada por

$$
L \varphi=\int_{0}^{r} d \eta(\theta) \varphi(-\theta),
$$

onde $\eta$ é uma função de variação limitada em $[0, r]$, normalizada tal que $\eta(0)=0$ e $\eta$ é contínua à direita em $(0, r)$, a valores no espaço de matrizes $\mathbb{R}^{n \times n}$. Este conjunto de funções é denotado $\mathrm{NBV} \stackrel{\text { def }}{=} \mathrm{NBV}\left([0, r], \mathbb{R}^{n \times n}\right)$. Podemos estender trivialmente $\eta \in \mathrm{NBV}$ em $\mathbb{R}$ fazendo $\eta(\theta)=0$ se $\theta<0$ e $\eta(\theta)=\eta(r)$ se $\theta>r$. Em (1.2), a notação $d \eta$ antes do integrando $\varphi$ enfatiza que $\eta$ é uma matriz e $\varphi$ é um vetor coluna, e portanto a integral é um vetor coluna. Quando tratar-se de uma família de operadores lineares $L(t)$, então para cada $t$ fica determinado $\eta(t, \cdot) \in \mathrm{NBV}$ e $L(t)$ fica escrita como

$$
L(t) \varphi=\int_{0}^{r} d \eta(t, \theta) \varphi(-\theta) .
$$

Um operador na forma (1.2) é dito atômico em 0 se o determinante da matriz $\eta(0-)$ é não nulo. Grosso modo, um operador é atômico em 0 quando 
há uma dependência explícita do valor de $\varphi(0)$ em $L \varphi$. No caso de famílias de operadores, como em (1.3), temos definições similares. Uma família $L(t)$ na forma (1.3) é dita uniformemente atômica em 0 se, para cada $\epsilon>0$, existir $\delta>0$ tal que

$$
\operatorname{Var}_{[0, \delta]} \eta(t, \cdot)-\eta(t, 0-)<\epsilon, \quad \forall t
$$

Seja $\Omega \subset \mathbb{R} \times \mathcal{C}$ um conjunto aberto e $D: \Omega \rightarrow \mathbb{R}^{n}$ contínua. Dizemos que $D$ é atômico em 0 se $D$ for Fréchet-diferenciável, junto com suas derivadas de primeira e segunda ordens, e se $D_{2}$ denota a derivada de $D$ com relação à segunda variável, então $D_{2}=D_{2}(t, \varphi)$ é atômico em 0 .

Seja $\Omega \subset \mathbb{R} \times \mathcal{C}$ um conjunto aberto. Dadas funções contínuas $D: \Omega \rightarrow \mathbb{R}^{n}$ e $f: \Omega \rightarrow \mathbb{R}^{n}$, sendo $D$ atômica em 0 , a relação

$$
\frac{d}{d t} D\left(t, x_{t}\right)=f\left(t, x_{t}\right)
$$

é dita uma equação diferencial funcional neutra, $\operatorname{EDFN}(D, f)$. A função $D$ é dita o operador diferença para a $\operatorname{EDFN}(D, f)$. Equações diferenciais da forma (1.4) $\operatorname{com} \mu=0$

$$
\dot{x}(t)=f\left(t, x_{t}\right), \quad x_{0}=\varphi
$$

são conhecidas como equações diferenciais funcionais retardadas (EDFR). Uma solução para o problema de valor inicial para a $\operatorname{EDFN}(D, f)$

$$
\begin{aligned}
\frac{d}{d t} D(t) x_{t} & =f\left(t, x_{t}\right) \\
x_{\sigma} & =\varphi
\end{aligned}
$$

é uma função $x(\cdot)=x(\cdot ; \sigma, \varphi)$ contínua e definida num intervalo $[\sigma-r, \sigma+A)$, para $A>0$, tal que $D(t) x_{t}$ seja continuamente diferenciável e as relações em (1.5) estão satisfeitas. Da mesma forma, dizemos que $x(\cdot)$ é uma solução que passa por $(\sigma, \varphi)$.

Se $D(t, \varphi)=D_{0}(t) \varphi+g(t)$ e $f(t, \varphi)=L(t) \varphi+h(t)$ onde $D_{0}(t)$ e $L(t)$ são lineares em $\varphi$ então, a $\operatorname{EDFN}(D, f)$ é dita linear. É linear homogênea se $g \equiv 0$ e $h \equiv 0$, caso contrário é dita linear não-homogênea. Se $D(t, \varphi)$ e $f(t, \varphi)$ não dependem de $t$, então a $\operatorname{EDFN}(D, f)$ é dita autônoma.

Como um exemplo, observamos que a equação diferencial linear

$$
\frac{d}{d t}[x(t)+C(t) x(t-1)]=A(t) x(t)+B(t) x(t-1), \quad t \geq 0,
$$


onde $A, B$ e $C$ são funções cujos valores são matrizes $n \times n$, pode ser escrita na forma (1.4) tomando-se $r=1, D(t) \varphi=\varphi(0)+C(t) \varphi(-1)$ e $f(t, \varphi)=$ $A(t) \varphi(0)+B(t) \varphi(-1)$. O operador $D(t)$ pode ser escrito na forma

$$
D(t)=\int_{0}^{r} d \mu(t, \theta) \varphi(-\theta)
$$

tomando $\mu(t, \theta)=0$ se $\theta \leqslant 0, \mu(\theta)=I$ se $0<\theta<1$ e $\mu(t, \theta)=I+C(t)$ para $\theta \geq 1$ (isto é, há "saltos" de tamanho $I$ e $C(t)$ respectivamente em $\theta=0$ e $\theta=1)$.

Como outro exemplo, sejam $r>0, x$ função real, $D \varphi=\varphi(0)-\varphi^{2}(-r)$ e $f: \Omega \rightarrow \mathbb{R}$ contínua, então o par $(D, f)$ define uma $E D F N$ dada pela equação

$$
\frac{d}{d t}\left[x(t)-x^{2}(t-r)\right]=f\left(t, x_{t}\right)
$$

\subsection{Teoria qualitativa}

Nesta seção enunciaremos sem demonstrações os teoremas básicos sobre existência, unicidade e dependência contínua com relação às condições iniciais. As demonstrações destes resultados podem ser encontradas em [6, cap. 2].

Teorema 1.1 (Existência e Unicidade) $S e \Omega$ é um aberto de $\mathbb{R} \times \mathcal{C} e(\sigma, \varphi) \in$ $\Omega$, então existe uma solução da $\operatorname{EDFN}(D, f)$ que passa por $(\sigma, \varphi)$. Além disso, se $f: \Omega \rightarrow \mathbb{R}^{n}$ for lipschitziana na segunda variável em subconjuntos compactos de $\Omega$, então, para qualquer $(\sigma, \varphi) \in \Omega$, existe uma única solução da $\operatorname{EDFN}(D, f)$ que passa por $(\sigma, \varphi)$.

Teorema 1.2 (Dependência Contínua) Sejam $\Omega \subset \mathbb{R} \times \mathcal{C}$ aberto, $\Lambda$ um subespaço de um espaço de Banach, e $D: \Omega \times \Lambda \rightarrow \mathbb{R}^{n}, f: \Omega \times \Lambda \rightarrow \mathbb{R}^{n}$ satisfazendo as seguintes hipóteses:

1. $D(t, \varphi, \lambda)$ é atômico em zero para cada $(t, \varphi) \in \Omega$ uniformemente com respeito a $\lambda$.

2. $D(t, \varphi, \lambda)$ e $f(t, \varphi)$ são contínuas em $(t, \varphi) \in \Omega$ para cada $\lambda \in \Lambda e$ também contínua em $\left(t, \varphi, \lambda_{0}\right)$ para $(t, \varphi) \in \Omega$.

3. $A \operatorname{EDFN}\left(D\left(\cdot, \lambda_{0}\right), f\left(\cdot, \lambda_{0}\right)\right)$ tem uma única solução definida num intervalo $[\sigma-r, b)$ que passa por $(\sigma, \varphi) \in \Omega$. 
Então existe uma vizinhança $N=N\left(\sigma, \varphi, \lambda_{0}\right)$ de $\left(\sigma, \varphi, \lambda_{0}\right)$ tal que para qualquer $\left(\sigma^{\prime}, \varphi^{\prime}, \lambda^{\prime}\right) \in N$, todas soluçôes $x\left(\sigma^{\prime}, \varphi^{\prime}, \lambda^{\prime}\right)$ da $\operatorname{EDFN}\left(D\left(\cdot, \lambda^{\prime}\right), f\left(\cdot, \lambda^{\prime}\right)\right)$ que passam por $\left(\sigma^{\prime}, \varphi^{\prime}\right)$ existem em $\left[\sigma^{\prime}-r, b\right]$ e $x_{t}\left(\sigma^{\prime}, \varphi^{\prime}, \lambda^{\prime}\right)$ é continua em $\left(t, \sigma^{\prime}, \varphi^{\prime}, \lambda_{0}\right)$ para $t \in[\sigma, \sigma+A] e\left[\sigma^{\prime}, \varphi^{\prime}, \lambda^{\prime}\right] \in N\left(\sigma, \varphi, \lambda_{0}\right)$.

Se $x$, definida num intervalo $[\sigma, a)$, é uma solução da $\operatorname{EDFN}(D, f)$, sendo $D$ e $f$ definidas em $\Omega \subset \mathbb{R} \times \mathcal{C}$, e se para cada $(\sigma, \varphi) \in \Omega$ existir uma única solução que passa por $(\sigma, \varphi)$, dizemos que $\hat{x}$ é continuação de $x$ se:

1. existe um $b>a$ tal que $\hat{x}$ é definido em $[\sigma-r, b)$,

2. $\hat{x}$ coincide $\operatorname{com} x$ em $[\sigma-r, a)$, e

3. $x$ satisfaz a $\operatorname{EDFN}(D, f)$ em $[\sigma, b)$.

Uma solução $x$ é não-continuável se tal continuação não existe. Neste caso, o intervalo $[\sigma, a)$ é então denominado intervalo maximal de existência da solução de $x$. A existência de uma solução não-continuável segue do bem conhecido Lema de Zorn. É conhecido que o intervalo maximal de existência é aberto.

Teorema 1.3 (Continuação) Suponhamos que $\Omega \subset \mathbb{R} \times \mathcal{C}$ seja aberto, $(D, f)$ define uma $\operatorname{EDFN}(D, f)$ em $\Omega, W \subset \Omega$ seja fechado e limitado, e que exista uma $\delta$-vizinhança de $W$ em $\Omega$. Se $f$ mapeia $W$ em um conjunto limitado de $\mathbb{R}^{n}, D(t, \varphi)$ e $D_{\varphi}(t, \varphi)$ (derivada em relação a $\varphi$ ) são uniformemente contínuas em $W, D$ é uniformemente atômico em 0 em $W$, e x é uma solução não-continuável da $\operatorname{EDFN}(D, f)$ em $[\sigma-r, b)$, então existe um $t^{\prime} \in[a, b)$ tal que $\left(t^{\prime}, x_{t^{\prime}}\right) \notin W$.

\subsection{Sistemas lineares gerais}

Para $(\sigma, \varphi) \in \mathbb{R} \times \mathcal{C}$, consideremos o sistema linear

$$
\left\{\begin{array}{l}
\frac{d}{d t}\left[D(t) x_{t}\right]=L(t) x_{t}+h(t), \quad t \geqslant \sigma, \\
x_{\sigma}=\varphi
\end{array}\right.
$$

onde $h \in L_{1}^{\text {loc }}$ e para todo $t \in \mathbb{R}, D(t): \mathcal{C} \rightarrow \mathbb{R}^{n}$ e $L(t): \mathcal{C} \rightarrow \mathbb{R}^{n}$ são dados por

$$
D(t) \varphi=\varphi(0)-\int_{-r}^{0} d[\mu(t, \theta)] \varphi(\theta), \quad L(t) \varphi=\int_{-r}^{0} d[\eta(t, \theta)] \varphi(\theta) .
$$


onde, por sua vez, os núcleos $\mu$ e $\eta$ satisfazem as seguintes afirmações.

i. O núcleo $\eta: \mathbb{R} \times \mathbb{R} \rightarrow \mathbb{R}^{n \times n},(t, \theta) \mapsto \eta(t, \theta)$, é mensurável em $(t, \theta)$ e para cada $t \in \mathbb{R}, \eta(t, \cdot) \in \mathrm{NBV}$. Além disso, existe um $m \in L_{1}^{\text {loc }}(\mathbb{R})$ tal que

$$
\operatorname{Var}_{[-r, 0]} \eta(t, \cdot) \leqslant m(t)
$$

ii. O núcleo $\mu: \mathbb{R} \times \mathbb{R} \rightarrow \mathbb{R}^{n \times n},(t, \theta) \mapsto \mu(t, \theta)$, é mensurável em $(t, \theta)$ e para cada $t \in \mathbb{R}, \mu(t, \cdot) \in \mathrm{NBV}$, sendo de variação limitada em $\theta$ no intervalo $[-r, 0]$ uniformemente em $t$, e tal que $t \mapsto D(t) \varphi$ é contínua para cada $\varphi$. Além disso, $\mu$ é uniformemente não-atômico em zero, isto é, dado $\epsilon>0$, existe um $\delta>0$ tal que

$$
\operatorname{Var}_{[-\delta, 0]} \mu(t, \cdot)<\epsilon, \quad \forall t \in \mathbb{R}
$$

Assim, temos o seguinte teorema que nos mostra a existência de uma única solução global.

Teorema 1.4 Sejam $\eta$ e $\mu$ como acima. Dados $\sigma \in \mathbb{R}, \varphi \in \mathcal{C}\left([-r, 0], \mathbb{R}^{n}\right)$, e $h \in L_{1}^{\text {loc }}\left([\sigma, \infty), \mathbb{R}^{n}\right)$, existe uma única função $x(\cdot ; \sigma, \varphi)$ definida continuamente em $[\sigma-r, \infty)$ que satisfaz o sistema $(1.6)$ em $[\sigma, \infty)$.

Para estes sistemas podemos exibir estimativas para o crescimento da solução. Este resultado encontra-se em [6], como Corolário 1.1 de seu nono capítulo.

Lema 1.5 Suponhamos que as hipóteses acima sobre $\eta, \mu$ e h estejam satisfeitas. Se $x(\cdot ; \sigma, \varphi, h)$ é a solução do sistema $(1.6)$ em $[\sigma, \infty)$ tal que $x_{\sigma}=\varphi$, então existem constantes positivas $C$ e $\gamma$ tais que

$$
|x(t ; \sigma, \varphi, h)| \leqslant C e^{\gamma t}\left(|\varphi|+\int_{\sigma}^{t}|h(s)| d s\right), \quad t \geqslant \sigma .
$$

\subsection{Operador solução para sistemas lineares}

Suponhamos que as condições do Teorema 1.1 para a unicidade de soluções estejam satisfeitas. Para qualquer $(\sigma, \varphi) \in \Omega$, existe um $t_{\sigma, \varphi}$ e uma função 
$x$ que é uma solução não-continuável da $\operatorname{EDFN}(D, f)$ em $\left[\sigma-r, t_{\sigma, \varphi}\right)$ que passa por $(\sigma, \varphi)$. Seja $\Omega_{\sigma} \subset \mathcal{C}$ definido por $\Omega_{\sigma}=\{\varphi \in \mathcal{C}:(\sigma, \varphi) \in \Omega\}$. Se falarmos somente de soluções não-continuáveis, podemos definir um operador $T(t, \sigma): \Omega_{\sigma} \rightarrow \mathcal{C}$ tal que para cada $\varphi \in \Omega_{s}$, e $t \in\left[\sigma, t_{\sigma, \varphi}\right)$

$$
T(t, \sigma) \varphi=x_{t}(\sigma, \varphi)
$$

Este operador será dito operador solução da $\operatorname{EDFN}(D, f)$. Queremos escrever o operador solução de uma EDFN como perturbação, por meio de um operador compacto, de um outro operador solução.

Consideremos a Equação Diferença (ED)

$$
D y_{t} 0, \quad t \geqslant 0
$$

onde $D$ é linear, contínuo e atômico em 0 . Se $\mathcal{C}_{D}=\{\varphi \in \mathcal{C}: D \varphi=0\}$, então a equação diferença (1.8) define um semigrupo de operadores lineares $T_{D}(t)$, $t \geqslant 0$, em $\mathcal{C}_{D}$, onde $T_{D}(t) \psi=y_{t}(\psi)$ com $y_{t}(\psi)$ sendo a solução de (1.8) que passa por $(0, \psi)$.

Seja $f: \mathcal{C} \rightarrow \mathbb{R}^{n}$ uma função contínua e $T_{D, f}(t)$ o semigrupo definido pela $\operatorname{EDFN}(D, f)$,

$$
\frac{d}{d t} D x_{t}=f\left(x_{t}\right), \quad t \geqslant 0
$$

Então podemos enunciar um teorema para a representação do semigrupo associado à equação (1.7), que se encontra em [6, Teo. 3.7.3].

Teorema 1.6 Sejam $D: \mathcal{C} \rightarrow \mathbb{R}^{n}$ linear, limitado e atômico em $0, \Phi=$ $\left(\varphi_{1}, \ldots, \varphi_{n}\right)$ uma n-upla de funções tal que $D \Phi=I$, e $\Psi=I-\Phi D$. Se $f$ é completamente continua e $T_{D, f}(t)$ é um operador limitado para cada $t>0$, então

$$
T_{D, f}(t)=T_{D}(t) \Psi+U(t)
$$

onde $U(t): \mathcal{C} \rightarrow \mathcal{C}, t \geqslant 0$, é compacto. 


\section{Capítulo 2}

\section{Sistemas Periódicos}

O propósito deste capítulo é apresentar a teoria geral para sistemas periódicos lineares com retardamento, e estendê-la a uma classe de sistemas periódicos para equações neutras.

\subsection{Sistemas periódicos com retardamento}

Esta primeira parte foi extraída do oitavo capítulo de Hale \& Verduyn Lunel [6] e apresenta uma teoria bastante análoga à teoria de Floquet para EDO. Estes autores argumentam que não é possível fazer de forma completa tal teoria (Floquet) para o caso das EDFR. Entretanto, é possível definir multiplicadores característicos e explorar a compacidade do operador solução para mostrar que a representação de Floquet existe no autoespaço generalizado de um multiplicador característico.

Suponhamos que $L: \mathbb{R} \rightarrow \mathcal{L}\left(\mathcal{C}, \mathbb{R}^{n}\right)$ satisfaça as condições da Seção 1.3 e que exista um $\omega>0$ tal que $L(t+\omega)=L(t)$ para todo $t$. Nesta seção, consideramos o sistema

$$
\dot{x}(t)=L(t) x_{t}
$$

e a extensão na qual existe uma teoria de Floquet.

Para qualquer $s \in \mathbb{R}, \varphi \in \mathcal{C}$, existe uma solução $x=x(s, \varphi)$ da equação $(2.1)$ definida em $[s, \infty)$ e $x_{t}(s, \varphi)$ é contínua em $t, s$ e $\varphi$. Seja $T(t, s) \varphi=$ $x_{t}(s, \varphi)$ para todo $t \geqslant s$ e $\varphi \in \mathcal{C}$. O operador $T(t, s)$ satisfaz a propriedade de semigrupo $T(t, s) T(s, \tau)=T(t, \tau)$ para todo $t \geqslant s \geqslant \tau$ e a periodicidade da Equação (2.1) implica que

$$
T(t+\omega, s)=T(t, s) T(s+\omega, s)
$$


para todo $t \geqslant s$. Seja $\Pi: \mathcal{C} \rightarrow \mathcal{C}$ o operador monodrômico definido por

$$
\Pi \varphi=T(\omega, 0) \varphi
$$

Seja $m$ o menor inteiro positivo tal que $m \omega \geqslant r$. Portanto, $\Pi^{m}=T(m \omega, 0)$ é um operador compacto, visto que no caso das EDFR, a solução ganha diferenciabilidade com o passar do tempo $t$. O teorema espectral polinomial e a teoria de operadores compactos implicam que o espectro $\sigma(\Pi)$ de $\Pi$ é um conjunto compacto contável do plano complexo com o único ponto de acumulação possível sendo 0 . Também, se $\mu \neq 0$ está em $\sigma(\Pi)$, então $\mu$ está no espectro pontual $\operatorname{P\sigma }(\Pi)$ de $\Pi$, isto é, existe um $\varphi \neq 0$ em $\mathcal{C}$ tal que $\Pi \varphi=\mu \varphi$. Todo $\mu \neq 0$ em $P \sigma(\Pi)$ é dito um multiplicador de Floquet ou multiplicador característico da equação $(2.1)$ e todo $\lambda$ tal que $\mu=e^{\lambda \omega}$ é dito expoente característico da equação (2.1).

Lema 2.1 $\mu=e^{\lambda \omega}$ é um multiplicador característico da equação (2.1) se, e só se, existe um $\varphi \neq 0$ em $\mathcal{C}$ tal que $T(t+\omega, 0) \varphi=\mu T(t, 0) \varphi$ para todo $t \geqslant 0$.

Demonstração: $(\Rightarrow)$ Se $\mu=e^{\lambda \omega} \in \mathbb{C}$, então existe um $\varphi \neq 0$ em $\mathcal{C}$ tal que $\Pi \varphi=\mu \varphi$. Fazendo $s=0$ em (2.2) temos

$$
T(t+\omega, 0) \varphi=\mu T(t, 0) \varphi
$$

para todo $t$.

$(\Leftarrow)$ Basta tomar $t=0$.

Como $\Pi^{m}$ é compacto (ver por exemplo [11]), para qualquer multiplicador característico $\mu$ de (2.1), existem dois subespaços $E_{\mu}$ e $Q_{\mu}$ de $\mathcal{C}$ tais que as seguintes propriedades são satisfeitas:

1. $E_{\mu}$ é de dimensão finita;

2. $E_{\mu} \oplus Q_{\mu}=\mathcal{C}$;

3. $\Pi E_{\mu} \subset E_{\mu}, \Pi Q_{\mu} \subset Q_{\mu}$;

4. $\sigma\left(\left.\Pi\right|_{E_{\mu}}\right)=\{\mu\}, \sigma\left(\left.\Pi\right|_{Q_{\mu}}\right)=\sigma(\Pi) \backslash\{\mu\}$.

A dimensão $d_{\mu}$ de $E_{\mu}$ é dita a multiplicidade do multiplicador $\mu$. Quando $d_{\mu}=1$, dizemos que $\mu$ é um autovalor simples. 
Seja $\Phi=\left\{\varphi_{1}, \ldots, \varphi_{d_{\mu}}\right\}$ uma base para $E_{\mu}$. Pela propriedade (3), $\Pi \Phi \subset$ $E_{\mu}$, então $\Pi \Phi$ pode ser escrito como combinação de $\Phi$ e assim existe uma matriz $d_{\mu} \times d_{\mu} M=M_{d_{\mu}}$ tal que $\Pi \Phi=\Phi M$, cujo único autovalor, pela propriedade (4), é $\mu \neq 0$. Portanto, existe uma matriz $d_{\mu} \times d_{\mu} B=B_{\mu}$ tal que $B=\omega^{-1} \ln M$. Definamos o vetor $P(t)$ com elementos em $\mathcal{C}$ por $P(t)=T(t, 0) \Phi e^{-B t}$. Então, para $t \geqslant 0$,

$$
\begin{aligned}
P(t+\omega) & =T(t+\omega) \Phi e^{-B(t+\omega)}=T(t, 0) T(w, 0) \Phi e^{-B(t+\omega)} \\
& =T(t, 0) \Pi \Phi e^{-B(t+\omega)}=T(t, 0) \Phi M e^{-B(t+\omega)} \\
& =T(t, 0) \Phi e^{B \omega} e^{-B \omega} e^{-B t}=T(t, 0) \Phi e^{-B t} \\
& =P(t) ;
\end{aligned}
$$

isto é, $P(t)$ é $\omega$-periódica. Podemos estender $P(t)$ periodicamente para $t \mathrm{em}$ $\mathbb{R}$. A função

$$
x_{t}(0, \Phi)=T(t, 0) \Phi=P(t) e^{B t}
$$

está bem definida para $t \in \mathbb{R}$ e cada coluna desta matriz é uma solução da equação (2.1) em $\mathbb{R}$. Em resumo, temos o seguinte resultado.

Lema 2.2 Se $\mu$ é um multiplicador característico da equação (2.1) e $\Phi$ é uma base para $E_{\mu}$ de dimensão $d_{\mu}$, existe uma matriz $d_{\mu} \times d_{\mu} B$ tal que $\sigma\left(e^{B \omega}\right)=$ $\{\mu\}$ e uma função matricial $n \times d_{\mu} P(t)$, com cada coluna em $\mathcal{C}, P(t+\omega)=$ $P(t), t \in \mathbb{R}$ tal que se $\varphi=\Phi b$ (combinação linear), então $x_{t}(\varphi)$ é definida para $t \in \mathbb{R} e$

$$
x_{t}(0, \varphi)=P(t) e^{B t} b .
$$

Portanto, em particular, $\mu=e^{\lambda \omega}$ é um multiplicador característico da equação (2.1) se, e só se, existe uma solução não-nula da equação (2.1) da forma

$$
x(t)=p(t) e^{\lambda t}
$$

onde $p(t+\omega)=p(t)$.

Para $\varphi \in E_{\mu}$, como $x_{t}(0, \varphi)(\theta)=x(0, \varphi)(t+\theta)=x_{t+\theta}(0, \varphi)(0),-r \leqslant \theta \leqslant$ 0 , segue que $P(t)(\theta)=x_{t}(0, \varphi)(\theta) e^{-B t}=x_{t+\theta}(0, \varphi)(0) e^{-B t}=P(t+\theta)(0) e^{B \theta}$, $-r \leqslant \theta \leqslant 0$. Portanto, se pormos $\tilde{P}(t+\theta)=P(t+\theta)(0)$, então $\Phi(\theta)=$ $x_{0}(0, \Phi)(\theta)=P(0)(\theta)=P(\theta)(0) e^{B \theta}=\tilde{P}(\theta) e^{B \theta} \mathrm{e}$

$$
x(0, \varphi)(t)=\tilde{P}(t) e^{B t} b, \quad t \in \mathbb{R}, \quad \varphi=\Phi b .
$$


Portanto, as soluções da Equação (2.1) com valor inicial em $E_{\mu}$ são de tipo Floquet, ou seja, se $\mu=e^{\lambda \omega}$, as soluções são da forma $e^{\lambda \omega}$ vezes um polinômio com coeficientes periódicos em $t$ de período $\omega$.

Também necessitamos da seguinte observação: Se $T(t, 0) \Phi b=0$ para algum $t$, então $b=0$. De fato, se existe um $t$ tal que $T(t, 0) \Phi b=0$ e $m \omega \geqslant t$, $m \geqslant 1$, então

$$
0=T(m \omega, 0) \Phi b=\Pi^{m} \Phi b=\Phi M^{n} b .
$$

Como o autovalor de $M^{n}$ é $\mu \neq 0$, então $b=0$.

Definimos os multiplicadores característicos da Equação (2.1) começando com tempo inicial 0. Para justificar esta escolha precisamos mostrar que os multiplicadores não dependem do tempo inicial. Para qualquer $s \in \mathbb{R}$, seja $\Pi(s)=T(s+\omega, s)$. Como antes, para todo $\mu \neq 0, \mu \in \sigma(\Pi(s))$, existem dois subespaços fechados $E_{\mu}(s)$ e $Q_{\mu}(s)$ de $\mathcal{C}$ tal que as propriedades (1)-(4) são válidas com as devidas mudanças de notação. Seja $\Phi(s)$ uma base para $E_{\mu}(s), \Pi(s) \Phi(s)=\Phi(s) M(s), \sigma(M(s))=\{\mu\}$. Como para o caso $s=0$, podemos definir $T(t, s) \Phi(s)$ para todo $t \in \mathbb{R}$. Para qualquer real $\tau$

$$
\begin{aligned}
\Pi(\tau) T(\tau, s) \Phi(s) & =T(\tau+\omega, \tau) T(\tau, s) \Phi(s) \\
& =T(\tau+\omega, s) \Phi(s) \\
& =T(\tau, s) T(s+\omega, s) \Phi(s) \\
& =T(\tau, s) \Phi(s) M(s) .
\end{aligned}
$$

Se $M=\mu I-N$, então $N$ é nilpotente e

$$
[\mu I-\Pi(\tau)] T(\tau, s) \Phi(s)=T(\tau, s) \Phi(s) N .
$$

Como $T(t, s) \Phi(s) b=0$ implica que $b=0$, segue que $\mu \in \sigma(\Pi(\tau))$ e a dimensão de $E_{\mu}(\tau)$ é pelo menos tão grande quanto a dimensão de $E_{\mu}(s)$. E como podemos inverter $s$ e $\tau$, obtemos o seguinte lema.

Lema 2.3 Os multiplicadores característicos da Equação (2.1) são independentes do tempo inicial e se $\Phi(s)$ é uma base para $E_{\mu}(s)$, então $T(t, s) \Phi(s)$ é uma base para $E_{\mu}(s)$ para todo $t \in \mathbb{R}$.

Sejam, agora,

$$
\begin{aligned}
\mathcal{Q}_{\mu} & =\bigcup_{t \in \mathbb{R}}\left(t, Q_{\mu}(t)\right), \\
\mathcal{E}_{\mu} & =\bigcup_{t \in \mathbb{R}}\left(t, E_{\mu}(t)\right) .
\end{aligned}
$$


então temos o lema seguinte, cuja demonstração pode ser encontrada em [6].

Lema 2.4 Para o sistema (2.1)

1. Os conjuntos $E_{\mu}(t)$ e $E_{\mu}(s)$ são difeomorfos $e$ os conjuntos $Q_{\mu}(s) e$ $Q_{\mu}(s)$ são homeomorfos para todo $t, s \in \mathbb{R}$.

2. O conjunto $\mathcal{E}_{\mu}$ em (2.4) é difeomorfo a $\mathbb{R} \times E_{\mu}(0)$ e o conjunto $\mathcal{Q}_{\mu}$ em (2.3) é homeomorfo a $\mathbb{R} \times Q_{\mu}(0)$.

3. Se $\frac{\partial}{\partial t} L(t, \varphi)$ é também contínua, então $\mathcal{Q}_{\mu}$ é difeomorfo a $\mathbb{R} \times Q_{\mu}(0)$. Estes difeomorfismos são definidos por $(\alpha, \varphi) \mapsto(\alpha, g(\alpha) \varphi), \alpha \in \mathbb{R}, \varphi \in$ $E_{\mu}(\alpha),(\alpha, \psi) \mapsto(\alpha, h(\alpha) \psi), \alpha \in \mathbb{R}, \psi \in Q_{\mu}(\alpha)$, onde $g$ e $h$ são continuamente diferenciáveis.

Notemos que se $\mu=e^{\lambda \omega}$ é simples, i.e. $d_{\mu}=1$, então $M(s)$, que é a matriz descrita anteriormente, referente ao tempo inicial $s$, é idêntica a $\mu$, $\Phi \stackrel{\text { def }}{=} \Phi(0)=\left\{\phi_{1}\right\}$ e $P(t)$ assume a forma simples

$$
e^{\lambda t} P(t)=T(t, 0) \varphi_{1}
$$

A projeção espectral sobre $E_{\mu}(s)$ ao longo de $Q_{\mu}(s)$ pode ser representada por uma integral de Dunford (Veja Gohberg, Goldberg \& Kaashoek [5])

$$
P_{\mu}(s)=\frac{1}{2 \pi i} \int_{\Gamma_{\mu}}(z I-\Pi(s))^{-1} d z
$$

onde $\Gamma_{\mu}$ é um círculo pequeno tal que $\mu$ é a única singularidade de $(z I-$ $\Pi(s))^{-1}$ dentro de $\Gamma_{\mu}$.

O próximo resultado relata a representação de Floquet em cada autoespaço da solução $x_{t}(s, \varphi)$ para qualquer $\varphi \in \mathcal{C}$. Suponhamos que $\sigma(\Pi)=$ $\{0\} \cup\left\{\mu_{m}\right\}$ onde $\left\{\mu_{m}\right\}$ é finito ou enumerável e cada $\mu_{m} \neq 0$. Para $\mu_{n} \in \sigma(\Pi)$, sejam $P_{n}(s): \mathcal{C} \rightarrow E_{\mu_{n}}(s), I-P_{n}(s): \mathcal{C} \rightarrow Q_{\mu_{n}}(s)$ as projeções induzidas por $E_{\mu_{n}}(t)$ e $Q_{\mu_{n}}(t)$ definidas anteriormente e satisfazendo as propriedades (1) $-(4)$.

Teorema 2.5 Sejam $\mu_{j}, j=1,2, \ldots$, os autovalores não-nulos do operador monodrômico $\Pi(s)$ ordenados por decrescimento de seus valores em módulo, e $\varphi \in \mathcal{C}$. Se $\gamma$ é um número real dado, então existem constantes positivas $\epsilon$ e $N$ tais que para $t \geqslant s$

$$
\left\|x_{t}(s, \varphi)-\sum_{\left|\mu_{n}\right|>e^{\gamma \omega}} P_{\mu_{n}}(s) x_{t}(s, \varphi)\right\| \leqslant N e^{(\gamma-\epsilon)(t-s)}\|\varphi\| .
$$


Demonstração: Seja $k=k(\gamma)$ um inteiro tal que $\left|\mu_{k}\right| \geqslant e^{\gamma \omega}$ e $\left|\mu_{k+1}\right|<e^{\gamma \omega}$, $\Sigma=\mu_{1}, \mu_{2}, \ldots, \mu_{k}$ e definimos

$$
E_{\Sigma}(s)=\bigoplus_{\mu \in \Sigma} E_{\mu}(s) \quad \text { e } \quad Q_{\Sigma}(s)=\bigcap_{\mu \in \Sigma} Q_{\mu}(s) .
$$

Então, temos $\mathcal{C}=E_{\Sigma} \oplus Q \Sigma$. Seja

$$
R_{k}(s) \varphi=\varphi-\sum_{j=1}^{k} P_{\mu_{j}}(s) \varphi .
$$

Pela invariância do operador solução $T(t, s)$, e como $R_{k}(s) \varphi \in Q_{\Sigma}(s)$, temos que $T(t, s) R_{k}(s) \varphi \in Q_{\Sigma}$ para todo $\varphi \in \mathcal{C}$. Além disso, existe uma constante $N_{0}$ tal que $\left\|R_{k}(s) \varphi\right\| \leqslant N_{0}\|\varphi\|$. Se $\epsilon$ é tal que $e^{(\gamma-2 \epsilon) \omega}=\left|\mu_{k+1}\right|$, então o raio espectral de

$$
\left.\widehat{\Pi}(s) \stackrel{\text { def }}{=} \Pi(s)\right|_{Q_{\Sigma}(s)}
$$

é $e^{(\gamma-2 \epsilon) \omega}$. Portanto, pela definição do raio espectral, $\lim _{n \rightarrow \infty}\left\|\widehat{\Pi}(s)^{n}\right\|^{1 / n}=$ $e^{(\gamma-2 \epsilon) \omega}$ e para algum $m>0$ temos

$$
\left\|\widehat{\Pi}(s)^{m} R_{k}(s) \varphi\right\| \leqslant e^{(\gamma-\epsilon) m \omega}\left\|R_{k}(s) \varphi\right\| .
$$

Como $\left.\widehat{T}(\tau, s) \stackrel{\text { def }}{=} T(\tau, s)\right|_{Q_{\Sigma}(s)}, s \leqslant \tau \leqslant s+\omega$, é um operador limitado para cada $\varphi$, pelo princípio da limitação uniforme, existe um $N_{1}$ tal que $\|\widehat{T}(\tau, s)\| \leqslant N_{1}$ para $s \leqslant \tau \leqslant s+\omega$. Seja $t \geqslant s$ dado e $k_{t}$ o maior inteiro tal que $s+k_{t} m \omega \leqslant t$, então

$$
\begin{aligned}
\left\|\widehat{T}(t, s) R_{k}(s) \varphi\right\| & =\left\|T\left(t, s+k_{t} m \omega\right) T\left(s+k_{t} m \omega, s\right) R_{k}(s) \varphi\right\| \\
& \leqslant m N_{1}\left\|\widehat{\Pi}(s)^{m}\right\|^{k_{t}}\left\|R_{k}(s) \varphi\right\| \\
& \leqslant m N_{1} e^{(\gamma-\epsilon) k_{t} \omega} N_{0}\|\varphi\| \\
& \leqslant N e^{(\gamma-\epsilon)(t-s)}\|\varphi\|,
\end{aligned}
$$

onde $N=m N_{1} N_{0}$. Isto prova a estimativa exponencial (2.7).

Corolário 2.6 A solução $x=0$ da Equação (2.1) é uniformemente assintoticamente estável se, e só se, todos os multiplicadores característicos da Equação (2.1) têm módulo menor do que 1. 
Demonstração: $(\Rightarrow)$ Segue do Teorema $2.5 \operatorname{com} \gamma=0$.

$(\Leftarrow)$ É consequência da representação de Floquet associado com qualquer multiplicador característico.

De maneira similar obtemos

Corolário 2.7 A solução $x=0$ da Equação (2.1) é uniformemente estável se, e só se, todos os multiplicadores característicos da equação (2.1) têm módulo menor ou igual a 1 . Além disso, se $\mu$ é um multiplicador com $|\mu|=1$, então todas as soluções da equação (2.1) com valor inicial em $E_{\mu}$ são limitadas.

Para operadores monodrômicos, a definição de dominância de um multiplicador característico é inspirada na condição $|\mu|>e^{\gamma \omega}$ (ver teorema 2.5). Assim, um autovalor $\mu_{d}$ de $\Pi$ é dito dominante se existir $\epsilon>0$ tal que

$$
\mu \in \sigma(\Pi) \text { e }|\mu|>\left|\mu_{d}\right|-\epsilon \Longrightarrow \mu=\mu_{d} .
$$

Esta definição de dominância é equivalente à das EDF autônomas, se considerarmos os expoentes característicos em vez dos multiplicadores característicos.

Teorema 2.8 Seja $\mu_{d}=e^{\lambda_{d} \omega}$ um autovalor simples e dominante de $\Pi(s)$. Se $P(t)$ é como em (2.5), então existem constantes positivas $N$ e $\epsilon$ tais que o comportamento assintótico da solução $x(t ; 0, \varphi)$ é dado por

$$
\left\|e^{-\lambda_{d} t} x_{t}(0, \varphi)-c(\varphi) P(t)\right\| \leqslant N e^{-\epsilon t}\|\varphi\|, \quad t \geqslant 0
$$

onde $c(\varphi)$ é definido de tal forma que $P_{\mu_{d}}(0) \varphi=c(\varphi) \varphi_{1}$.

Demonstração: Se $\mu=e^{\lambda \omega}$ é um autovalor simples de $\Pi$, como $T(t, s)$ mapeia difeomorficamente $E_{\mu_{d}}(s)$ em $E_{\mu_{d}}(t)$, segue da equação (2.5) que

$$
\begin{aligned}
P_{\mu_{d}}(t) x_{t}(0, \varphi) & =P_{\mu_{d}} T(t, 0) \varphi \\
& =T(t, 0) P_{\mu_{d}}(0) \varphi \\
& =c(\varphi) e^{\lambda_{d} t} P(t) .
\end{aligned}
$$

Portanto, o resultado segue do Teorema 2.5 


\subsection{Sistemas periódicos neutros}

Estes resultados têm generalizações naturais para EDFN. Consideremos a EDFN linear periódica

$$
\frac{d}{d t} D(t) x_{t}=L(t) x_{t}
$$

onde $D(t)$ e $L(t)$ são famílias, contínuas em $t$, de operadores lineares contínuos, e existe $\omega>0$ tal que $D(t+\omega)=D(t), L(t+\omega)=L(t)$ para todo $t$. Além disso, $D(t)$ é atômico em zero. Seja $T_{D, L}(t, \sigma), t \geqslant \sigma$, o operador solução da equação (2.9). Um número complexo $\rho$ é dito um multiplicador característico da $\operatorname{EDFN}(2.9)$ se $\rho$ é um autovalor de tipo finito de $T_{D, L}(\sigma+\omega, \sigma)$, isto é, é um ponto isolado do espectro de $T_{D, L}(\sigma+\omega, \sigma)$, cujo autoespaço generalizado tem dimensão finita. Como antes, os multiplicadores característicos são independentes de $\sigma$. Para cada multiplicador característico $\rho$ e cada $\sigma \in \mathbb{R}$, existe uma decomposição $\mathcal{C}=E_{\sigma} \oplus Q_{\sigma}$, onde $E_{\sigma}=\mathcal{N}\left(T_{D, L}(\sigma+\omega, \sigma)-\rho I\right)^{k}, Q_{\sigma}=\mathcal{R}\left(T_{D, L}(\sigma+\omega, \sigma)-\rho I\right)^{k}$, e $E_{\sigma}$ tem dimensão finita $d$. Se $\Phi_{\sigma}$ é uma base para $E_{\sigma}$, então existem matrizes $B_{\sigma} \mathrm{e}$ $C_{\sigma}(t)$, respectivamente $d \times d$ e $n \times n$, sendo $B_{\sigma}$ constante, o espectro de $e^{B_{\sigma} \omega}$ sendo o conjunto $\{\rho\}$, e $C_{\sigma}(t+\omega)=C_{\sigma}(t)$. Então para todo $\varphi=\Phi_{\sigma} b \in E_{\sigma}$, temos que

$$
T_{D, L}(t+\sigma, \sigma)=C_{\sigma}(t) e^{B_{\sigma} t} b
$$

Desta forma, existe uma representação de Floquet no autoespaço generalizado $E_{\sigma}$ do multiplicador característico $\rho$.

A representação dada pelo Teorema 1.6 é válida para a equação (2.9). De fato, podemos determinar uma constante $k$ tal que para qualquer $\sigma \in \mathbb{R}$, existe um $\Phi^{\sigma}=\left(\varphi_{1}^{\sigma}, \ldots, \varphi_{n}^{\sigma}\right) \operatorname{com} D(\sigma) \Phi^{\sigma}=I$, e $\left|\varphi_{j}^{\sigma}\right| \leqslant k, j=1,2, \ldots, n$. Se $\Psi^{\sigma}=I-\Phi^{\sigma} D(\sigma)$, então

$$
T_{D, L}(t, \sigma)=T_{D}(t, \sigma) \Psi^{\sigma}+U(t, \sigma),
$$

onde $U(t, \sigma)$ é compacto para $t \geqslant \sigma$ e $T_{D}(t, \sigma)$ é o operador solução da equação diferença $D(t) y_{t}=0$.

Usando o mesmo tipo de raciocínio como para o caso autônomo, se $e^{a_{D} \omega}$ é o raio espectral de $T_{D}(\sigma+\omega, \sigma)$, pode-se mostrar que qualquer $\rho$ no espectro de $T_{D}(\sigma+\omega, \sigma) \operatorname{com}|\rho|>e^{a_{D} \omega}$ é um autovalor normal e, assim, um multiplicador característico da equação (2.9). Além disso, existe somente um número finito de multiplicadores característicos $\rho$ satisfazendo $|\rho|>e^{a \omega}$ para todo 
$a>a_{D}$. Neste caso, o espaço $\mathcal{C}$ pode ser decomposto como $\mathcal{C}=E_{\sigma, a} \oplus Q_{\sigma, a}$, onde $E_{\sigma, a}$ e $Q_{\sigma, a}$ são invariantes sob $T_{D}(\sigma+\omega, \sigma)$, o espectro de $\left.T_{D}(\sigma+\omega)\right|_{E_{\sigma, a}}$ consiste somente em multiplicadores $\rho$ tais que $|\rho| \geqslant e^{a \omega}$, o espectro de $\left.T_{D}(\sigma+\omega)\right|_{Q_{\sigma, a}}$ fica contido no disco de centro em 0 e raio $e^{(a-\epsilon) \omega}$ para algum $\epsilon>0$. Portanto, existe uma constante $K$ tal que

$$
\left|T_{D, L}(t, \sigma)\right| Q_{\sigma, a} \mid \leqslant K e^{(a-\epsilon)(t-\sigma)}, \quad t \geqslant \sigma .
$$

Da mesma forma, existem matrizes $B_{\sigma, a}$ e $C_{\sigma, a}(t)$, sendo $B_{\sigma, a}$ constante e $C_{\sigma, a}(t) \omega$-periódica, tais que

$$
T_{D, L}(t+\sigma, \sigma) \Phi_{\sigma, a}=C_{\sigma, a}(t) e^{B_{\sigma, a} t}, \quad t \in \mathbb{R},
$$

onde $\Phi_{\sigma, a}$ é uma base para $E_{\sigma, a}$ e os únicos autovalores de $e^{B_{\sigma, a} \omega}$ são os multiplicadores característicos $\rho$ da equação (2.9) $\operatorname{com}|\rho| \geqslant e^{a \omega}$.

Para terminar o capítulo, enunciamos dois resultados apresentados na seção anterior, cujas provas são idênticas, bastando ressaltar que, enquanto no caso das EDFR, é automático que todo elemento não nulo do espectro é do tipo finito, e que há sempre um número finito destes cujo módulo ultrapassa qualquer valor, isto deve ser pedido por hipótese no caso neutro. Notamos que os mesmos conceitos de projeção espectral, como apresentados nas equações (2.5) e (2.6), estendem-se naturalmente para o caso das EDFN. A definição de autovalor dominante encontra-se em (2.8).

Teorema 2.9 Sejam $\mu_{j}, j=1,2, \ldots$, os autovalores não-nulos do operador monodrômico $\Pi(s)$ ordenados por decrescimento de seus valores em módulo, $e \varphi \in \mathcal{C}$. Se $\gamma$ é um número real dado tal que existe um número finito de autovalores de $\Pi(s)$ tais que seus módulos ultrapassem $e^{\gamma \omega}$, então existem constantes positivas $\epsilon$ e $N$ tais que para $t \geqslant s$

$$
\left\|x_{t}(s ; \varphi)-\sum_{\left|\mu_{n}\right|>e^{\gamma \omega}} P_{\mu_{n}}(s) x_{t}(s ; \varphi)\right\| \leqslant N e^{(\gamma-\epsilon)(t-s)}\|\varphi\| .
$$

Teorema 2.10 Seja $\mu_{d}=e^{\lambda_{d} \omega}$ um autovalor simples e dominante de $\Pi(s)$. Se $P(t)$ é como em (2.5), então existem constantes positivas $N$ e $\epsilon$ tais que o comportamento assintótico da solução $x(t ; 0, \varphi)$ é dado por

$$
\left\|e^{-\lambda_{d} t} x_{t}(0, \varphi)-c(\varphi) P(t)\right\| \leqslant N e^{-\epsilon t}\|\varphi\|, \quad t \geqslant 0
$$

onde $c(\varphi)$ é definido de tal forma que $P_{\mu_{d}}(0) \varphi=c(\varphi) \varphi_{1}$. 


\section{Capítulo 3}

\section{Uma Classe de Sistemas Periódicos}

Neste capítulo estudaremos com mais profundidade o comportamento assintótico de uma classe de equações diferenciais funcionais, para a qual fomos capazes de calcular explicitamente o resolvente de um operador monodrômico, obtendo as informações necessárias sobre seu espectro. Fomos capazes de demonstrar vários resultados da teoria espectral de forma independente, e obtivemos um resultado sobre estimativas precisas para o comportamento assintótico das soluções destas equações, tendo por hipótese o conhecimento da dominância de um expoente característico. Estes resultados, em combinação com resultados sobre a dominância de raízes de equações características em [4], nos permitem obter resultados inéditos que estendem resultados em $[3,8]$.

\subsection{Teoria espectral}

Consideremos a seguinte equação linear neutra periódica

$$
\frac{d}{d t}\left(x(t)+\sum_{i=1}^{k} c_{i} x(t-i \omega)\right)=a(t) x(t)+\sum_{i=1}^{k} b_{i}(t) x(t-i \omega)
$$

onde as funções $a(t)$ e $b_{i}(t), i=1,2, \ldots, k$, são contínuas e $\omega$-periódicas, $c_{i}$, $i=0,1, \ldots, k$, (assumimos $c_{0}=1$ ) são números reais, não necessariamente não nulos. Notamos que os retardamentos são múltiplos do período $\omega$. Nestas condições, tomamos por espaço de fase o conjunto $\mathcal{C}=\mathcal{C}([-k \omega, 0], \mathbb{R})$. Usando a mudança de variáveis abaixo, podemos eliminar o termo $a(t) x(t)$ da equação (3.1). 
Lema 3.1 Seja $A=\frac{1}{\omega} \int_{0}^{\omega} a(s) d s$. Pela mudança de variáveis

$$
y(t)=\exp \left(-\int_{t_{0}}^{t} a(s) d s\right) x\left(t+t_{0}\right),
$$

a equação (3.1), sujeita a condição inicial $x_{t_{0}}=\varphi$ pode ser reescrita como o problema de valor inicial

$$
\begin{aligned}
& \frac{d}{d t}\left[y(\tau)+\sum_{i=1}^{k} c_{i} e^{-i \omega A} y(\tau-i \omega)\right]= \\
& \sum_{i=1}^{k}\left(b_{i}\left(\tau-t_{0}\right)-c_{i} a\left(\tau-t_{0}\right)\right) e^{-i \omega A} y(\tau-i \omega) .
\end{aligned}
$$

sujeito à condição $y_{0}=\hat{\varphi}$, com

$$
\hat{\varphi}(\theta)=\exp \left(\int_{\theta}^{t_{0}} a(s) d s\right) \varphi(\theta)
$$

Demonstração: Temos que, para $t \geqslant 0$,

$$
\begin{aligned}
x(t) & +\sum_{i=1}^{k} c_{i} x(t-i \omega) \\
& =y\left(t-t_{0}\right) e^{\left(\int_{t_{0}}^{t} a(s) d s\right)}+\sum_{i=1}^{k} c_{i} y\left(t-t_{0}-i \omega\right) e^{\left(\int_{t_{0}}^{t-i \omega} a(s) d s\right)} \\
& =\left[y\left(t-t_{0}\right)+\sum_{i=1}^{k} c_{i} y\left(t-t_{0}-i \omega\right) e^{\left(-\int_{t-i \omega}^{t} a(s) d s\right)}\right] e^{\left(\int_{t_{0}}^{t} a(s) d s\right)}
\end{aligned}
$$

Pela periodicidade de $a(t), \int_{t-n \omega}^{t} a(s) d s=-n \int_{0}^{\omega} a(s) d s$. Assim

$$
=\left[y\left(t-t_{0}\right)+\sum_{i=1}^{k} c_{i} e^{-i \omega A} y\left(t-t_{0}-i \omega\right)\right] e^{\left(\int_{t_{0}}^{t} a(s) d s\right)}
$$


Também,

$$
\begin{aligned}
\sum_{i=1}^{k} b_{i}(t) x(t-i \omega) & =\sum_{i=1}^{k} b_{i}(t) y\left(t-t_{0}-i \omega\right) e^{\left(\int_{t_{0}}^{t-i \omega} a(s) d s\right)} \\
& =\left[\sum_{i=1}^{k} b_{i}(t) y\left(t-t_{0}-i \omega\right) e^{\left(-\int_{t-i \omega}^{t} a(s) d s\right)}\right] e^{\left(\int_{t_{0}}^{t} a(s) d s\right)} \\
& =\left[\sum_{i=1}^{k} b_{j}(t) e^{-i \omega A} y\left(t-t_{0}-i \omega\right)\right] e^{\left(\int_{t_{0}}^{t} a(s) d s\right)}
\end{aligned}
$$

Finalmente, de (3.1), usando (3.4), (3.5) e fazendo $\tau=t-t_{0}$, obtemos que

$$
\begin{aligned}
& 0=\frac{d}{d t}\left[x(t)+\sum_{i=1}^{k} c_{i} x(t-i \omega)\right]-a(t) x(t)-\sum_{i=1}^{k} b_{i}(t) x(t-i \omega) \\
& =\frac{d}{d t}\left[\left(y\left(t-t_{0}\right)+\sum_{i=1}^{k} c_{i} e^{-i \omega A} y\left(t-t_{0}-i \omega\right)\right) e^{\left(\int_{t_{0}}^{t} a(s) d s\right)}\right] \\
& +\left(-a(t) y\left(t-t_{0}\right)-\sum_{i=1}^{k} b_{i}(t) y\left(t-t_{0}-i \omega\right)\right) e^{\left(\int_{t_{0}}^{t} a(s) d s\right)} \\
& =\left[\frac{d}{d t}\left(y(\tau)+\sum_{i=1}^{k} c_{i} e^{-i \omega A} y(\tau-i \omega)\right)\right. \\
& +a\left(\tau+t_{0}\right)\left(y(\tau)+\sum_{i=1}^{k} c_{i} e^{-i \omega A} y(\tau-i \omega)\right) \\
& \left.-a\left(\tau+t_{0}\right) y(\tau)-\sum_{i=1}^{k} b_{i}(t) y(\tau-i \omega)\right] e^{\left(\int_{t_{0}}^{\tau+t_{0}} a(s) d s\right)} \\
& =\left[\frac{d}{d t}\left(y(\tau)+\sum_{i=1}^{k} c_{i} e^{-i \omega A} y(\tau-i \omega)\right)+a\left(\tau+t_{0}\right) \sum_{i=1}^{k} c_{i} e^{-i \omega A} y(\tau-i \omega)\right. \\
& \left.-\sum_{i=1}^{k} e^{-i \omega A} b_{i}\left(\tau+t_{0}\right) y(\tau-i \omega)\right] e^{\left(\int_{t_{0}}^{\tau+t_{0}} a(s) d s\right)} .
\end{aligned}
$$

Assim, encontrar $x$ que satisfaça (3.1) é equivalente a encontrar uma solução $y$ de (3.3). 
Portanto podemos supor, sem perda de generalidade, que $a(t)=0 \mathrm{em}$ (3.1), sendo suficiente analisar a equação

$$
\frac{d}{d t}\left(x(t)+\sum_{i=1}^{k} c_{i} x(t-i \omega)\right)=\sum_{j=1}^{k} b_{j}(t) x(t-j \omega) .
$$

\subsection{0 operador monodrômico}

Seja $\Pi(s): \mathcal{C} \rightarrow \mathcal{C}$ o operador monodrômico $\Pi(s)=T(s+\omega, s)$, i.e.,

$$
(\Pi(s) \varphi)=x_{s+\omega}(\cdot ; s, \varphi)
$$

Como o comportamento assintótico das soluções é independente do tempo inicial $s$, podemos colocar $s=0$ e definir $\Pi=\Pi(0)$. Integrando a equação diferencial (3.6) e usando a periodicidade de $b_{j}$, temos a seguinte representação para $\Pi$.

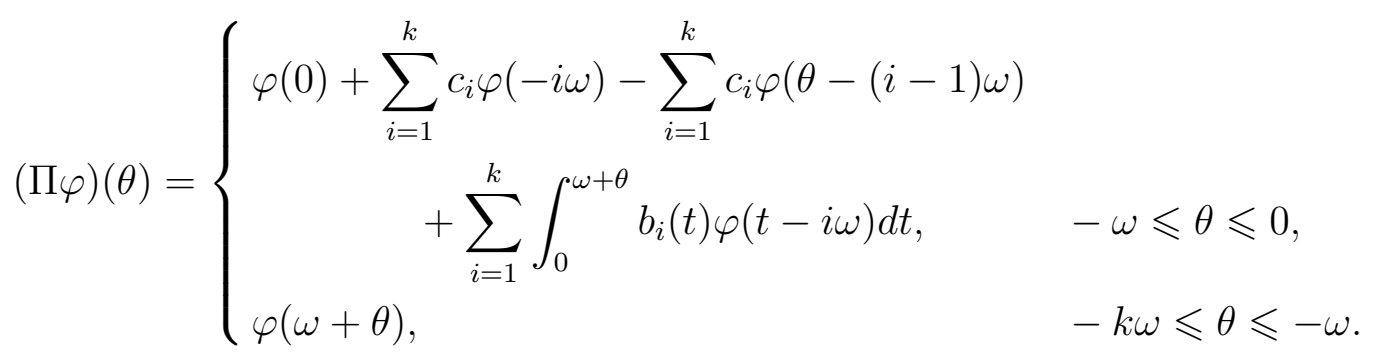

Podemos calcular explicitamente o resolvente de $\Pi$.

Teorema 3.2 O resolvente $(z I-\Pi)^{-1}$ do operador monodrômico $\Pi$ é dado por

$$
\begin{aligned}
& \quad(z I-\Pi)^{-1} \varphi(\theta)=\Omega_{-\omega}^{\theta}(1 / z)\left[z I-\Omega_{-\omega}^{0}\right]^{-1}\left(\varphi(-\omega)+G_{\varphi, z}(0)\right)+G_{\varphi, z}(\theta), \\
& \text { para }-\omega \leqslant \theta \leqslant 0 e
\end{aligned}
$$$$
(z I-\Pi)^{-1} \varphi(\theta)=\frac{1}{z^{m}}(z I-\Pi)^{-1} \varphi(\theta+m \omega)+\sum_{j=0}^{m-1} \frac{\varphi(\theta+j \omega)}{z^{j+1}}
$$ 


$$
\begin{aligned}
& \text { para }-\omega \leqslant \theta+m \omega \leqslant 0, m=1, \ldots, k-1, \text { onde } \\
& \qquad \begin{aligned}
G_{\varphi, z}(\theta)=\frac{z^{k-1}}{C_{z}}\left\{\varphi(\theta)-\Omega_{-\omega}^{\theta}\left(\frac{1}{z}\right) \varphi(-\omega)\right. \\
\quad-\sum_{i=2}^{k} \sum_{j=1}^{i-1} \frac{c_{i}}{z^{i-j}}\left[\varphi(\theta-j \omega)-\Omega_{-\omega}^{\theta}\left(\frac{1}{z}\right) \varphi(-(j+1) \omega)\right] \\
\left.+\sum_{i=2}^{k} \sum_{j=1}^{i-1} \int_{-\omega}^{\theta} \Omega_{\sigma}^{\theta}\left(\frac{1}{z}\right) \frac{b_{i}(\sigma)}{z^{i-j}} \varphi(\sigma-j \omega) d \sigma\right\} \\
+\frac{z^{2 k-1}}{C_{z}^{2}}\left\{\sum_{l=1}^{k} \frac{1}{z^{l}} \int_{-\omega}^{\theta} b_{l}(\sigma) \Omega_{\sigma}^{\theta}\left(\frac{1}{z}\right) \varphi(\sigma) d \sigma\right. \\
\left.\quad-\sum_{i=2}^{k} \sum_{l=1}^{k} \sum_{j=1}^{i-1} c_{i} z^{j-i-l} \int_{-\omega}^{\theta} b_{l}(\sigma) \Omega_{\sigma}^{\theta}\left(\frac{1}{z}\right) \varphi(\sigma-j \omega) d \sigma\right\},
\end{aligned}
\end{aligned}
$$

em que $C_{z}$ é o polinômio (lembrar que $c_{0}=1$ )

$$
C_{z}=\sum_{i=0}^{k} c_{i} z^{k-i}
$$

$e \Omega_{s}^{t}=\Omega_{s}^{t}\left(\frac{1}{z}\right)$ é dado por

$$
\Omega_{s}^{t}\left(\frac{1}{z}\right)=\exp \left(\sum_{i=1}^{k} \frac{z^{k-i}}{C_{z}} \int_{s}^{t} b_{i}(\theta) d \theta\right) .
$$

Demonstração: Dado $\varphi \in \mathcal{C}$, suponha que $(z I-\Pi)^{-1} \varphi=\psi$. Então

$$
\varphi=z \psi-\Pi \psi \text {. }
$$

Suponhamos a princípio que $\varphi$ seja diferenciável. Da representação de $\Pi$ segue o sistema de equações

$$
\begin{array}{cr}
\varphi(\theta)=z \psi(\theta)-\psi(\theta+\omega), & -k \omega \leqslant \theta \leqslant-\omega \\
\dot{\varphi}(\theta)=z \dot{\psi}(\theta)+\sum_{i=1}^{k} c_{i} \dot{\psi}(\theta-(i-1) \omega) & -\omega \leqslant \theta \leqslant 0 \\
\quad-\sum_{i=1}^{k} b_{i}(\theta) \psi(\theta-(i-1) \omega) & \\
\varphi(-\omega)=z \psi(-\omega)-\psi(0) &
\end{array}
$$


Usando (3.12) indutivamente, para $l=1,2, \ldots, k-1$ e $-\omega \leqslant \theta \leqslant 0$, e derivando em relação a $\theta$, obtemos

$$
\begin{aligned}
& \psi(\theta-l \omega)=\frac{1}{z^{l}} \psi(\theta)+\sum_{j=1}^{l} \frac{1}{z^{l-j+1}} \varphi(\theta-j \omega) \\
& \dot{\psi}(\theta-l \omega)=\frac{1}{z^{l}} \dot{\psi}(\theta)+\sum_{j=1}^{l} \frac{1}{z^{l-j+1}} \dot{\varphi}(\theta-j \omega)
\end{aligned}
$$

Substituindo (3.15) e (3.16) em (3.13) e usando a fórmula de $C_{z}$ dada em (3.10), vem que

$$
\begin{aligned}
\dot{\varphi}(\theta)=z \dot{\psi}(\theta) & +\sum_{i=1}^{k} c_{i} \dot{\psi}(\theta-(i-1) \omega)-\sum_{i=1}^{k} b_{i}(\theta) \psi(\theta-(i-1) \omega) \\
=z \dot{\psi}(\theta) & +\sum_{i=1}^{k} c_{i}\left[\frac{1}{z^{i-1}} \dot{\psi}(\theta)+\sum_{j=1}^{i-1} \frac{1}{z^{i-j}} \dot{\varphi}(\theta-j \omega)\right] \\
& -\sum_{i=1}^{k} b_{i}(\theta)\left[\frac{1}{z^{i-1}} \psi(\theta)+\sum_{j=1}^{i-1} \frac{1}{z^{i-j}} \varphi(\theta-j \omega)\right] \\
=\frac{C_{z}}{z^{k-1}} \dot{\psi}(\theta) & +\sum_{i=1}^{k} \sum_{j=1}^{i-1} \frac{c_{i}}{z^{i-j}} \dot{\varphi}(\theta-j \omega) \\
& -\sum_{i=1}^{k} b_{i}(\theta)\left[\frac{1}{z^{i-1}} \psi(\theta)+\sum_{j=1}^{i-1} \frac{1}{z^{i-j}} \varphi(\theta-j \omega)\right]
\end{aligned}
$$

Portanto, obtemos a equação diferencial ordinária linear

$$
\begin{aligned}
& \dot{\psi}(\theta)-\sum_{i=1}^{k} \frac{z^{k-i}}{C_{z}} b_{i}(\theta) \psi(\theta) \\
& \quad=\frac{z^{k-1}}{C_{z}}\left[\dot{\varphi}(\theta)-\sum_{i=1}^{k} \sum_{j=1}^{i-1} \frac{c_{i}}{z^{i-j}} \dot{\varphi}(\theta-j \omega)+\sum_{i=1}^{k} \sum_{j=1}^{i-1} \frac{b_{i}(\theta)}{z^{i-j}} \varphi(\theta-j \omega)\right]
\end{aligned}
$$

Consideremos a parte homogênea da EDO (3.17),

$$
\dot{\psi}(\theta)-\sum_{i=1}^{k} \frac{z^{k-i}}{C_{z}} b_{i}(\theta) \psi(\theta)=0 .
$$

Seja $\Omega_{s}^{t}\left(\frac{1}{z}\right)$ a solução fundamental da $\operatorname{EDO}(3.18)$, i.e., $\psi(t)=\Omega_{s}^{t}\left(\frac{1}{z}\right) \psi(s)$ é uma solução de (3.18) e $\Omega_{s}^{s}\left(\frac{1}{z}\right)=1$. Podemos calcular $\Omega_{s}^{t}\left(\frac{1}{z}\right)$ explicitamente, obtendo a fórmula (3.11). Da fórmula das variações das constantes, obtemos

$$
\psi(\theta)=\Omega_{-\omega}^{\theta}\left(\frac{1}{z}\right) \psi(-\omega)+G_{\varphi, z}(\theta)
$$


onde

$$
\begin{aligned}
G_{\varphi, z}(\theta)=\frac{z^{k-1}}{C_{z}} \int_{\omega}^{\theta} \Omega_{\sigma}^{\theta}\left(\frac{1}{z}\right)\left[\dot{\varphi}(\sigma)-\sum_{i=1}^{k}\right. & \sum_{j=1}^{i-1} \frac{c_{i}}{z^{i-j}} \dot{\varphi}(\sigma-j \omega) \\
& \left.+\sum_{i=1}^{k} \sum_{j=1}^{i-1} \frac{b_{i}(\sigma)}{z^{i-j}} \varphi(\sigma-j \omega)\right] d \sigma
\end{aligned}
$$

Temos que

$$
\frac{d}{d \theta} \Omega_{s}^{\theta}\left(\frac{1}{z}\right)=\left[\sum_{i=1}^{k} \frac{z^{k-i}}{C_{z}} b_{i}(\theta)\right] \Omega_{s}^{\theta}\left(\frac{1}{z}\right), \quad \frac{d}{d \theta} \Omega_{\theta}^{s}\left(\frac{1}{z}\right)=-\left[\sum_{i=1}^{k} \frac{z^{k-i}}{C_{z}} b_{i}(\theta)\right] \Omega_{\theta}^{s}\left(\frac{1}{z}\right),
$$

visto que $\Omega_{s}^{\theta}\left(\frac{1}{z}\right)$ é solução de (3.18) e $\Omega_{\theta}^{s}\left(\frac{1}{z}\right)=1 / \Omega_{s}^{\theta}\left(\frac{1}{z}\right)$. Agora, integrando por partes,

$$
\begin{aligned}
\int_{-\omega}^{\theta} \Omega_{\sigma}^{\theta}\left(\frac{1}{z}\right) \dot{\varphi}(\sigma-a) d \sigma=\varphi( & \theta-a)-\Omega_{-\omega}^{\theta}\left(\frac{1}{z}\right) \varphi(-\omega-a) \\
& +\sum_{i=1}^{k} \frac{z^{k-i}}{C_{z}} \int_{-\omega}^{\theta} b_{i}(\sigma) \Omega_{\sigma}^{\theta}\left(\frac{1}{z}\right) \varphi(\sigma-a) d \sigma
\end{aligned}
$$

Usando (3.22), podemos eliminar a derivada $\dot{\varphi}$ de (3.20), e empregando (3.21), obtemos a fórmula em (3.9).

Falta ainda obtermos $\psi(-\omega)$ em (3.19). Fazendo $\theta=0$ em (3.19) e usando a condição de fronteira (3.14), obtemos

$$
\varphi(-\omega)=\left(z-\Omega_{-\omega}^{0}\left(\frac{1}{z}\right)\right) \psi(-\omega)+G_{\varphi, z}(0)
$$

donde

$$
\psi(-\omega)=\left[z I-\Omega_{-\omega}^{0}\right]^{-1}\left(\varphi(-\omega)-G_{\varphi, z}(0)\right)
$$

Usando (3.23) em (3.19), obtemos (3.7). De (3.15) obtem-se (3.8). Para finalizar a prova, notemos que a expressão para $(z I-\Pi)^{-1} \varphi$, na fórmula (3.7), é bem definida para $\varphi \in \mathcal{C}$ e podemos abandonar a suposição de que $\varphi$ seja diferenciável, usando o fato de que o conjunto das funções diferenciáveis é denso em $\mathcal{C}$. 


\subsection{Comportamento assintótico}

A representação para o resolvente de $\Pi$ nos fornece informações importantes sobre as propriedades espectrais do operador. Temos que

$$
\sigma(\Pi) \backslash\{0\}=\left\{z \in \mathbb{C} \mid\left(z-\Omega_{-\omega}^{0}\left(\frac{1}{z}\right)\right)=0 \quad \text { ou } \quad C_{z}=0\right\}
$$

Segue que todo elemento não nulo do espectro é isolado, por ser zero de funções inteiras não nulas. Da representação (3.7), tendo em vista a fórmula para $\Omega_{-\omega}^{\theta}\left(\frac{1}{z}\right)$ em (3.11), se $z_{0} \in \sigma(\Pi) \backslash\{0\}$ é tal que $C_{z_{0}}=0$, há uma singularidade essencial em $(z I-\Pi)^{-1}$ para $z=z_{0}$, e não temos a decomposição espectral em autoespaços generalizados de dimensão finita, como discutido no capítulo 2. No caso em que $z_{0} \in \sigma(\Pi) \backslash\{0\}$ e $C_{z_{0}} \neq 0$, é fácil ver que $z=z_{0}$ é um pólo (de ordem finita) do resolvente $(z I-\Pi)^{-1}$. De [11, Teo. V.10.1] segue a existência da decomposição espectral em autoespaços generalizados de dimensão finita, que é a imagem da projeção espectral que discutimos a seguir. Quando o autoespaço generalizado associado a um multiplicador característico $\mu$ é unidimensional, dizemos que $\mu$ é simples.

Além disso, usando a representação de Dunford da projeção espectral $P_{\mu}$ de $\Pi$ sobre um autoespaço generalizado $E_{\mu}, \mu \in \sigma(\Pi) \backslash\{0\}$,

$$
P_{\mu}=\operatorname{Res}_{z=\mu}(z I-\Pi)^{-1}
$$

podemos calcular explicitamente a projeção espectral de П usando o cálculo de resíduos. O lema a seguir nos dá uma condição suficiente para um multiplicador característico seja simples e uma fórmula explícita para a projeção espectral sobre o autoespaço generalizado unidimensional.

Lema 3.3 Seja $\mu \in \sigma(\Pi) \backslash\{0\}$ tal que $C_{\mu} \neq 0$, onde $C_{\mu}$ é dado em (3.10), e que $\mu$ seja um zero simples da equação

$$
z-\Omega_{-\omega}^{0}\left(\frac{1}{z}\right)=0
$$

Então o autoespaço generalizado $E_{\mu}$ é unidimensional, gerado pela função $\varphi_{1}(\theta)=\Omega_{-\omega}^{\theta}\left(\frac{1}{\mu}\right)$, e a projeção espectral $P_{\mu}: \mathcal{C} \rightarrow E_{\mu}$ é dada por

$$
P_{\mu} \varphi=\left(\left[\left.\frac{d}{d z}\left(z-\Omega_{-\omega}^{0}\left(\frac{1}{z}\right)\right)\right|_{z=\mu}\right]^{-1}\left(\varphi(-\omega)+G_{\varphi, \mu}(0)\right)\right) \varphi_{1}
$$


Demonstração: Seja $\mu$ tal que $C_{\mu} \neq 0$ e tal que $\mu-\Omega_{-\omega}^{0}\left(\frac{1}{\mu}\right)=0$. Se $\mu$ é simples então a função

$$
z \mapsto(z-\mu)\left[z-\Omega_{-\omega}^{0}\left(\frac{1}{z}\right)\right]^{-1}
$$

é analítica numa vizinhança de $z=\mu \mathrm{e}$

$$
\begin{aligned}
\lim _{z \rightarrow \mu}(z-\mu)\left[z-\Omega_{-\omega}^{0}\left(\frac{1}{z}\right)\right]^{-1} & =\lim _{z \rightarrow \mu}\left(\frac{z-\Omega_{-\omega}^{0}\left(\frac{1}{z}\right)}{z-\mu}\right)^{-1} \\
& =\lim _{z \rightarrow \mu}\left(\frac{z-\Omega_{-\omega}^{0}\left(\frac{1}{z}\right)-\left(\mu-\Omega_{-\omega}^{0}\left(\frac{1}{\mu}\right)\right)}{z-\mu}\right)^{-1} \\
& =\left[\left.\frac{d}{d z}\left(z-\Omega_{-\omega}^{0}\left(\frac{1}{z}\right)\right)\right|_{z=\mu}\right]^{-1}
\end{aligned}
$$

Como $C_{\mu} \neq 0$, as expressões $G_{\varphi, z}$ e $\Omega_{-\omega}^{\theta}\left(\frac{1}{z}\right)$, dadas respectivamente por (3.9) e (3.11), são analíticas em $z=\mu$. De (3.25), e da fórmula explícita para o resolvente de $\Pi$ dada por (3.7)-(3.8), e da discussão acima, vem que a função

$$
z \mapsto(z-\mu)[z I-\Pi]^{-1}
$$

é analítica numa vizinhança de $z=\mu$.

Por $\mu$ ser simples, para $\theta \in[-\omega, 0]$, de (3.25) e (3.28), temos

$$
\begin{aligned}
P_{\mu} \varphi(\theta) & =\lim _{z \rightarrow \mu}(z-\mu)(z I-\Pi)^{-1} \varphi(\theta) \\
& =\lim _{z \rightarrow \mu}(z-\mu)\left(\Omega_{-\omega}^{\theta}(1 / z)\left[z-\Omega_{-\omega}^{0}\right]^{-1}\left(\varphi(-\omega)+G_{\varphi, z}(0)\right)+G_{\varphi, z}(\theta)\right) \\
& =\Omega_{-\omega}^{\theta}\left(\frac{1}{\mu}\right)\left[\left.\frac{d}{d z}\left(z-\Omega_{-\omega}^{0}\left(\frac{1}{z}\right)\right)\right|_{z=\mu}\right]^{-1}\left(\varphi(-\omega)+G_{\varphi, \mu}(0)\right)
\end{aligned}
$$

Agora estudaremos $P_{\mu} \varphi(\theta)$ no caso $-\omega \leqslant \theta+m \omega \leqslant 0, m=1, \ldots, k-1$. Da hipótese em que as funções $b_{j}(t)$ são $\omega$-periódicas, de (3.11), segue que $\Omega_{s}^{t}(1 / z)$ satisfaz

$$
\Omega_{s}^{t}(1 / z)\left(\frac{1}{\mu}\right)=\Omega_{s+j \omega}^{t+j \omega}\left(\frac{1}{\mu}\right), \quad j \in \mathbb{Z} .
$$

Daí,

$$
\begin{aligned}
\Omega_{-w}^{\theta+m \omega}\left(\frac{1}{\mu}\right) & =\underbrace{\Omega_{-\omega}^{0}\left(\frac{1}{\mu}\right)}_{\mu} \underbrace{\Omega_{0}^{\omega}\left(\frac{1}{\mu}\right)}_{\mu} \underbrace{\Omega_{\omega}^{2 \omega}\left(\frac{1}{\mu}\right)}_{\mu} \cdots \underbrace{\Omega_{(m-2) \omega}^{(m-1) \omega}\left(\frac{1}{\mu}\right)}_{\mu} \Omega_{-\omega+m \omega}^{\theta+m \omega}\left(\frac{1}{\mu}\right) \\
& =\mu^{m} \Omega_{-\omega}^{\theta}\left(\frac{1}{\mu}\right) .
\end{aligned}
$$


Temos também que a expressão $\sum_{j=0}^{m-1} \frac{\varphi(\theta+j \omega)}{z^{j+1}}$, em (3.8), é analítica em $z=\mu$. De (3.8), (3.29) e (3.30), obtemos

$$
\begin{aligned}
P_{\mu} \varphi(\theta) & =\lim _{z \rightarrow \mu}(z-\mu)(z I-\Pi)^{-1} \varphi(\theta) \\
& =\lim _{z \rightarrow \mu}(z-\mu)\left[\frac{1}{z^{m}}(z I-\Pi)^{-1} \varphi(\theta+m \omega)+\sum_{j=0}^{m-1} \frac{\varphi(\theta+j \omega)}{z^{j+1}}\right] \\
& =\frac{1}{\mu^{m}} \lim _{z \rightarrow \mu}(z-\mu)(z I-\Pi)^{-1} \varphi(\theta+m \omega) \\
& =\frac{1}{\mu^{m}} \Omega_{-\omega}^{\theta+m \omega}\left(\frac{1}{\mu}\right)\left[\left.\frac{d}{d z}\left(z-\Omega_{-\omega}^{0}\left(\frac{1}{z}\right)\right)\right|_{z=\mu}\right]^{-1}\left(\varphi(-\omega)+G_{\varphi, \mu}(0)\right) \\
& =\Omega_{-\omega}^{\theta}\left(\frac{1}{\mu}\right)\left[\left.\frac{d}{d z}\left(z-\Omega_{-\omega}^{0}\left(\frac{1}{z}\right)\right)\right|_{z=\mu}\right]^{-1}\left(\varphi(-\omega)+G_{\varphi, \mu}(0)\right)
\end{aligned}
$$

Notemos que as fórmulas (3.29) e (3.31) são a mesma, eliminando qualquer restrição sobre $\theta$. Como $E_{\mu}$ é unidimensional e $P_{\mu}$ tem imagem em $E_{\mu}$, seguem as considerações sobre $\varphi_{1}$ e a fórmula (3.27).

A seguir apresentamos resultados sobre o comportamento assintótico das soluções, como aplicação dos teoremas 2.9 e 2.10. A definição de autovalor dominante encontra-se em (2.8), na página 27.

Teorema 3.4 Se $\mu_{d}$ é um autovalor simples e dominante de $\Pi$, então o comportamento assintótico da solução $x(\cdot)$ de (3.6), sujeita à condição inicial $x_{0}=\varphi$, é dado por

$$
\lim _{t \rightarrow \infty} \Omega_{t}^{-\omega}\left(\frac{1}{\mu}\right) x(t ; 0, \varphi)=\left[\left.\frac{d}{d z}\left(z-\Omega_{-\omega}^{0}\left(\frac{1}{z}\right)\right)\right|_{z=\mu}\right]^{-1}\left(\varphi(-\omega)+G_{\varphi, \mu}(0)\right) .
$$

Demonstração: Pelo Lema 3.3, temos que $c(\varphi)$, usado no Teorema 2.10, página 29 , é dado por

$$
c(\varphi)=\left[\left.\frac{d}{d z}\left(z-\Omega_{-\omega}^{0}\left(\frac{1}{z}\right)\right)\right|_{z=\mu}\right]^{-1}\left(\varphi(-\omega)+G_{\varphi, \mu}(0)\right) .
$$

Temos que

$$
\frac{d}{d t} \Omega_{-\omega}^{t}\left(\frac{1}{\mu}\right)=\left[\sum_{j=1}^{k} \frac{\mu^{k-j}}{C_{\mu}} b_{j}(t)\right] \Omega_{-\omega}^{t}\left(\frac{1}{\mu}\right) .
$$


e, de (3.30), para $i$ inteiro,

$$
\Omega_{-\omega}^{t-i \omega}\left(\frac{1}{\mu}\right)=\frac{1}{\mu^{i}} \Omega_{-\omega}^{t}\left(\frac{1}{\mu}\right) .
$$

Agora se tomamos

$$
x(t)=\Omega_{-\omega}^{t}\left(\frac{1}{\mu}\right),
$$

então, usando (3.34) e (3.35) e a periodicidade das funções $b_{j}$, vem que

$$
\begin{aligned}
\frac{d}{d t}(x(t) & \left.+\sum_{i=1}^{k} c_{i} x(t-i \omega)\right)-\sum_{j=1}^{k} b_{j}(t) x(t-j \omega) \\
& =\frac{d}{d t}\left(\Omega_{-\omega}^{t}\left(\frac{1}{\mu}\right)+\sum_{i=1}^{k} c_{i} \Omega_{-\omega}^{t-i \omega}\left(\frac{1}{\mu}\right)\right)-\sum_{i=1}^{k} b_{j}(t) \Omega_{-\omega}^{t-i \omega}\left(\frac{1}{\mu}\right) \\
& =\left(\sum_{i=0}^{k} c_{i} \mu^{k-i} \sum_{j=1}^{k} \frac{\mu^{-j}}{C_{\mu}} b_{j}(t)-\sum_{i=1}^{k} b_{j}(t) \frac{1}{\mu^{i}}\right) \Omega_{-\omega}^{t}\left(\frac{1}{\mu}\right)=0 .
\end{aligned}
$$

Portanto $x(t)$ é solução de (3.6), na página 34, e satisfaz a condição inicial $x_{0}=\varphi_{1}$. Logo

$$
\left[T(t, 0) \varphi_{1}\right](\theta)=\Omega_{-\omega}^{t+\theta}\left(\frac{1}{\mu}\right)
$$

e do Lema 2.2, na página 23 e de (2.5), na página 25 , vem que a função $P(t)$ a valores em $\mathcal{C}$, dada por

$$
[P(t)](\theta)=e^{-\mu t} \Omega_{-\omega}^{t+\theta}\left(\frac{1}{\mu}\right)
$$

é $\omega$-periódica, e como é contínua e dada por uma exponencial, satisfaz

$$
0<c_{1} \leqslant[P(t)](\theta) \leqslant c_{2}
$$

para certas constantes positivas $c_{1}$ e $c_{2}$. Seque que

$$
\frac{1}{[P(t)](0)}=\frac{1}{e^{-\mu t} \Omega_{-\omega}^{t}\left(\frac{1}{\mu}\right)} \leqslant \frac{1}{c_{1}} .
$$

Da estimativa fornecida pelo Teorema 2.10, página 29, usando que $|\psi(0)|<$ $\|\psi\|$ e (3.38), obtemos a seguinte seqüência de implicações

$$
\begin{gathered}
\exists \epsilon>0, \quad\left\|e^{-\mu t} x_{t}(0, \varphi)-c(\varphi) P(t)\right\| \leqslant N e^{-\epsilon t}\|\varphi\|, \quad \forall t \geqslant 0 \\
\Longrightarrow \lim _{t \rightarrow \infty} e^{-\mu t} x(t ; 0, \varphi)-c(\varphi) e^{-\mu t} \Omega_{-\omega}^{t}\left(\frac{1}{\mu}\right)=0 \\
\Longrightarrow \lim _{t \rightarrow \infty} \Omega_{t}^{-\omega}\left(\frac{1}{\mu}\right) x(t ; 0, \varphi)=c(\varphi)
\end{gathered}
$$

Da última relação e de (3.33), segue (3.32). 


\section{Capítulo 4}

\section{Algumas Aplicações}

\subsection{Um exemplo estudado}

Consideremos como primeiro exemplo a equação diferencial

$$
\frac{d}{d t}[x(t)-x(t-1)]=\cos ^{2}(2 \pi t) x(t-1)
$$

Podemos tomar o período $\omega$ como 1. Estamos interessados em estudar o comportamento assintótico de soluções de (4.1), aplicando os resultados das seções anteriores. Para começar, calculamos o espectro não nulo do operador monodrômico, dado pela fórmula (3.24). É necessário que calculemos $\Omega_{t}^{s}$, que é a solução fundamental de

$$
\dot{y}-\frac{\cos ^{2}(2 \pi t)}{(z-1)} y=0
$$

Resolvemos esta equação para obtermos

$$
\Omega_{0}^{t}=\exp \left[\frac{1}{z-1}\left(\frac{t}{2}+\frac{\operatorname{sen}(4 \pi t)}{8 \pi}\right)\right] .
$$

Daí, temos

$$
\Omega_{-1}^{0}=\frac{1}{\Omega_{0}^{-1}}=\exp \left[\frac{1}{2(z-1)}\right]
$$

De (4.1) tiramos que

$$
C_{z}=z-1
$$


Portanto, de (3.24), (4.2) e (4.3), obtemos que o espectro do operador monodrômico para a EDFN periódica (4.1) é

$$
\sigma(\Pi) \backslash\{0\}=\left\{\mu \in \mathbb{C} \mid\left(\mu-\exp \left[\frac{1}{2(\mu-1)}\right]\right)=0 \quad \text { ou } \quad \mu=1\right\}
$$

Analisemos os zeros da equação característica

$$
z-\exp \left[\frac{1}{2(z-1)}\right]=0
$$

Fazendo $\mu=e^{\lambda}$

$$
e^{\lambda}=\exp \left[\frac{1}{2\left(e^{\lambda}-1\right)}\right] \Leftrightarrow \lambda-2 k \pi i=\frac{1}{2\left(e^{(\lambda-2 k \pi i)}-1\right)}
$$

Portanto, sem perda de generalidade, podemos analisar os zeros $\mu$ de (4.5) através da equação (por expoentes característicos)

$$
\lambda=\frac{1}{2\left(e^{\lambda}-1\right)}
$$

que equivale a

$$
2 \lambda\left(e^{\lambda}-1\right)-1=0
$$

Usando métodos simples do Cálculo, obtemos que a função $x \mapsto f(x)=$ $\frac{1}{2\left(e^{x}-1\right)}$ é estritamente decrescente, $\lim _{x \rightarrow-\infty} f(x)=-1 / 2, \lim _{x \rightarrow 0-} f(x)=$ $-\infty, \lim _{x \rightarrow 0+} f(x)=+\infty$ e $\lim _{x \rightarrow \infty} f(x)=0$. Como a função identidade é crescente, segue que existem duas raízes reais, uma positiva, à qual chamamos $\lambda_{0}$, e outra negativa. O valor de $\lambda_{0}$ é aproximadamente 0,6034 . O expoente característico $\lambda_{0}$ é simples pois

$$
\frac{d}{d \lambda}\left[2 \lambda\left(e^{\lambda}-1\right)-1\right]_{\lambda=\lambda_{0}}=\frac{1}{\lambda_{0}}+2 \lambda_{0} e^{\lambda_{0}}>0 .
$$

A seguir, provaremos que o expoente característico $\lambda_{0}$ é dominante. Suponha que $\lambda=a+b i$ seja outra raiz de (4.7) tal que $a>0$. Sem perda de generalidade, podemos supor $b \geqslant 0$. Tomando as partes real e imaginária em (4.7), obtemos o sistema

$$
\begin{gathered}
2 a e^{a} \cos b-2 b e^{a} \operatorname{sen} b=2 a+1 \\
2 a e^{a} \operatorname{sen} b+2 b e^{a} \cos b=2 b
\end{gathered}
$$


Fazendo $a(4.8 \mathrm{a})+b(4.8 \mathrm{~b}) \mathrm{e}-b(4.8 \mathrm{a})+a(4.8 \mathrm{~b})$, temos

$$
\begin{gathered}
2 e^{a}\left(a^{2}+b^{2}\right) \cos b=a+2\left(a^{2}+b^{2}\right) \\
2 e^{a}\left(a^{2}+b^{2}\right) \operatorname{sen} b=-b
\end{gathered}
$$

Fazendo, agora $((4.9 \mathrm{a}))^{2}+((4.9 \mathrm{~b}))^{2}$

$$
\begin{aligned}
4 e^{2 a}\left(a^{2}+b^{2}\right)^{2} & =\left(a+2\left(a^{2}+b^{2}\right)\right)^{2}+b^{2} \\
& =a^{2}+4\left(a^{2}+b^{2}\right) a+4\left(a^{2}+b^{2}\right)^{2}+b^{2} \\
\Longrightarrow \quad 4 e^{2 a} & =\frac{\left(a^{2}+b^{2}\right)(1+4 a)+4\left(a^{2}+b^{2}\right)^{2}}{\left(a^{2}+b^{2}\right)^{2}} \\
& =\frac{1+4 a}{a^{2}+b^{2}}+4
\end{aligned}
$$

De (4.10) obtemos uma relação entre $a$ e $b$. Suponhamos que $b \geqslant \frac{\pi}{4}$ e analisemos (4.10) em busca de $a$. Assim

$$
4 e^{2 a} \leqslant \frac{1+4 a}{a^{2}+\frac{\pi^{2}}{16}}+4=: h(a)
$$

Utilizando as ferramentas do Cálculo, mostra-se que, em $[0, \infty)$, a função $h(\cdot)$ tem um máximo global para $x_{\text {máx }}=\left(\sqrt{1+\pi^{2}}-1\right) / 4$ e portanto

$$
h(x) \leqslant h\left(x_{\text {máx }}\right)=\frac{4\left(1+\pi^{2}+\sqrt{1+\pi^{2}}\right)}{1+\pi^{2}-\sqrt{1+\pi^{2}}}, \quad x \in[0, \infty) .
$$

De (4.11) e (4.12) vem que

$$
a \leqslant \frac{1}{2} \ln \left(\frac{1+\pi^{2}+\sqrt{1+\pi^{2}}}{1+\pi^{2}-\sqrt{1+\pi^{2}}}\right) \approx 0,313<\lambda_{0}
$$

Logo, se $\lambda$ é raiz de (4.7) com $|\operatorname{Im} \lambda| \geqslant \pi / 4$, então $\operatorname{Re} \lambda<\lambda_{0}$. Suponhamos agora, que $\lambda=a+b i$ é raiz de (4.7) tal que $0<b \leqslant \frac{\pi}{4}$ (temos sen $b>0$ e $\cos b>0)$. Multiplicando (4.8b) por $\exp \left(\frac{b \cos b}{\operatorname{sen} b}\right) / 2 \operatorname{sen} b$, obtemos

$$
\left(a+\frac{b \cos b}{\operatorname{sen} b}\right) \exp \left(a+\frac{b \cos b}{\operatorname{sen} b}\right)=\frac{b}{\operatorname{sen} b} \exp \left(\frac{b \cos b}{\operatorname{sen} b}\right) .
$$

Usando a função $W$ de Lambert, veja (A.21), página 55, verifica-se facilmente que $x=W(x) e^{W(x)}$ implica

$$
W^{\prime}(x)=\frac{1}{x}\left(1-\frac{1}{1+W(x)}\right) .
$$


Aplicando estas considerações a (4.13), vem que

$$
a=-\frac{b \cos b}{\operatorname{sen} b}+W\left(\frac{b}{\operatorname{sen} b} e^{\left(\frac{b \cos b}{\operatorname{sen} b}\right)}\right):=g(b)
$$

que define $a$ como função de $b, 0<b<\pi / 4$. Queremos mostrar que, para $0<b<\pi / 4$, teremos $a<\lambda_{0}$. Para tanto, calculamos a derivada de $g(\cdot)$ na fórmula (4.15).

$$
g^{\prime}(b)=\frac{-\operatorname{sen} b \cos b+b}{\operatorname{sen}^{2} b}+W^{\prime}\left(\frac{b}{\operatorname{sen} b} e^{\left(\frac{b \cos b}{\operatorname{sen} b}\right)}\right) \frac{\operatorname{sen}^{2} b-b^{2}}{\operatorname{sen}^{3} b} e^{\left(\frac{b \cos b}{\operatorname{sen} b}\right)}
$$

Multiplicando ambos os lados por sen $b>0$, usando (4.14), a abreviação $W=$ $W\left(\frac{b}{\operatorname{sen} b} \exp \left(\frac{b \cos b}{\operatorname{sen} b}\right)\right)>0$, as relações ${ }^{1}(\operatorname{sen} b-b \cos b)>0$ e $\left(b^{2}-\operatorname{sen}^{2} b\right)>0$, temos

$$
\begin{aligned}
g^{\prime}(b) \operatorname{sen} b & =-\cos b+\frac{b}{\operatorname{sen} b}+\frac{\operatorname{sen} b}{b} e^{\left(\frac{-b \cos b}{\operatorname{sen} b}\right)} e^{\left(\frac{b \cos b}{\operatorname{sen} b}\right)}\left(1-\frac{1}{1+W}\right) \frac{\operatorname{sen}^{2} b-b^{2}}{\operatorname{sen}^{2} b} \\
& =\frac{-b \operatorname{sen} b \cos b+b^{2}+\operatorname{sen}^{2} b-b^{2}+\frac{1}{1+W}\left(b^{2}-\operatorname{sen}^{2} b\right)}{b \operatorname{sen} b} \\
& =\frac{\operatorname{sen} b(\operatorname{sen} b-b \cos b)+\frac{1}{1+W}\left(b^{2}-\operatorname{sen}^{2} b\right)}{b \operatorname{sen} b}>0
\end{aligned}
$$

Portanto $g(b)$ é estritamente crescente para $0<b<\pi / 4$. Vem que

$$
a<g(\pi / 4)=-\frac{\pi}{4}+W\left(\frac{\pi \sqrt{2}}{4} e^{\pi / 4}\right) \approx 0,16<\lambda_{0}
$$

Logo, o expoente característico $\lambda_{0}$ é dominante, assim como o multiplicador característico $\mu_{0}=e^{\lambda_{0}}$.

Estamos em posição de aplicarmos o Teorema 3.4. Dada uma $\varphi \in \mathcal{C}$, o

\footnotetext{
${ }^{1}$ Para $b>0$ temos $(\operatorname{sen} b-b \cos b)>0$ pois $\tan b>b$ e $\left(b^{2}-\operatorname{sen}^{2} b\right)>0$ pois $b>\operatorname{sen} b$.
} 
comportamento assintótico da solução $x(t ; 0, \varphi)$ de $(4.1)$ é dado por

$$
\begin{aligned}
\lim _{t \rightarrow \infty} \exp \left(\frac{1}{1-\mu_{0}}\left[\frac{1+t}{2}+\frac{\operatorname{sen}(4 \pi t)}{8 \pi}\right]\right) x(t ; 0, \varphi) \\
=\frac{2\left(\mu_{0}-1\right)^{2}}{2\left(\mu_{0}-1\right)^{2}+\exp \left(\left[2\left(\mu_{0}-1\right)\right]^{-1}\right)} \times \\
\quad\left[\varphi(-1)+\frac{\varphi(0)}{\mu_{0}-1}-\exp \left(\frac{1}{2\left(\mu_{0}-1\right)}\right) \frac{\varphi(-1)}{\mu_{0}-1}\right. \\
\left.\quad+\frac{1}{\left(\mu_{0}-1\right)^{2}} \int_{-1}^{0} \cos ^{2}(2 \pi s) \exp \left(\frac{1}{1-\mu_{0}}\left[\frac{s}{2}+\frac{\operatorname{sen}(4 \pi s)}{8 \pi}\right]\right) d s\right] .
\end{aligned}
$$

Fazemos ainda a observação de que, com relação à equação (4.1), não seria possível derivar o comportamento assintótico das soluções utilizando-se apenas os resultados contidos em Philos \& Purnaras [8], visto que não há zero $\lambda_{0}$ (necessariamente deveria ser um zero dominante da equação característica nos expoentes equivalente a $(4.7))$ que satisfaz a hipótese $\left(\mathrm{P}\left(\lambda_{0}\right)\right)$ do Teorema 1 de [8], semelhante à condição (A.19) ne página 54, que implica a dominância de um determinado autovalor, sob certas condições estritas.

\subsection{Exemplo sem autoespaços de dimensão finita}

Seja $f: \mathbb{R} \rightarrow \mathbb{R}$ uma função contínua 1-periódica tal que $\int_{0}^{1} f(t) d t=0$. Considere a equação diferencial periódica

$$
\frac{d}{d t}[x(t)-x(t-1)]=f(t) x(t-1) .
$$

Seja $\Omega_{t}^{s}$ a solução fundamental de

$$
\dot{y}(t)=\frac{1}{(z-1)} f(t) y(t) .
$$

Então

$$
\Omega_{t}^{s}\left(\frac{1}{z}\right)=\exp \left(\frac{\int_{s}^{t} f(s) d s}{(z-1)}\right),
$$

e a equação característica da Equação (4.16) é dada por

$$
z-\Omega_{-1}^{0}\left(\frac{1}{z}\right)=z-1=0 \quad \Longleftrightarrow \quad z=1 .
$$


Como em (4.16), $c_{1}=-1$ e $k=1$, por (3.10) temos

$$
C_{z}=z-1
$$

Logo, $\sigma(\Pi) \backslash\{0\}=\{1\}$. Mas $z=1$, sendo zero de $C_{z}$, faz com que $(z I-\Pi)^{-1}$ tenha uma singularidade essencial em $z=1$. Como discutido na página 38 , não podemos aplicar a teoria aqui apresentada para calcular o comportamento assintótico das soluções de (4.16).

\subsection{Certas equações periódicas simples}

Estamos interessados em estudar o comportamento assintótico das soluções do problema de valor inicial

$$
\begin{gathered}
\dot{x}(t)=a(t) x(t)+b(t) x(t-1), \\
x_{t_{0}}=\varphi
\end{gathered}
$$

onde $a(t)$ e $b(t)$ são funções reais contínuas 1-periódicas. Pelo Lema 3.1, página 32 , pelas mudanças de variáveis

$$
y(t)=\exp \left(\int_{t}^{t_{0}} a(s) d s\right) x\left(t+t_{0}\right), \quad \hat{\varphi}(\theta)=e^{\left(\int_{\theta}^{t_{0}} a(s) d s\right)} \varphi(\theta)
$$

temos que (4.18) é equivalente ao seguinte PVI

$$
\begin{gathered}
\dot{y}(t)=b(t) e^{-A} y(t-1) \\
x_{0}=\hat{\varphi}
\end{gathered}
$$

onde $A=\int_{-1}^{0} a(s) d s$. Da representação (3.11) temos

$$
\Omega_{s}^{t}\left(\frac{1}{z}\right)=\exp \left(\frac{e^{-A}}{z} \int_{s}^{t} b(\theta) d \theta\right)
$$

pois em (4.20), $k=1$ e $C_{z}=z$. Fazendo $B=\int_{-1}^{0} b(\theta) d \theta$ e $D=e^{-A} B$, obtemos

$$
\Omega_{-1}^{0}\left(\frac{1}{z}\right)=e^{D / z}
$$

Assim a equação característica de (4.20) é dada por

$$
z-e^{D / z}=0 \Longleftrightarrow z=e^{D / z} .
$$


Fazendo $z=e^{\lambda}$ obtemos

$$
e^{\lambda}=e^{D / e^{\lambda}} \Longleftrightarrow \lambda-2 k \pi=\frac{D}{e^{\lambda}}=\frac{D}{e^{\lambda-2 k \pi}},
$$

sendo suficiente analisar a equação

$$
\lambda-D e^{-\lambda}=0
$$

Se $-e^{-1}<D$, então, pelo Lema A.3, página 55, $\lambda_{0}=W(D)=W\left(e^{-A} B\right)$ é simples e dominante, onde a função $W$ é dada em (A.21) na Página 55. Em particular, se $B>-e^{-1}$ e $B=-A$, então $\lambda_{0}=W\left(B e^{B}\right)=B$. Pelo Lema 3.3, vem que o autoespaço generalizado unidimensional associado a $\mu_{0}=e^{\lambda_{0}}$ é gerado pela função $\varphi_{1}(\theta)=\Omega_{-1}^{\theta}\left(\frac{1}{z}\right)$. Temos então, pelo Teorema 3.4, página 40 , que o comportamento assintótico da solução $y(\cdot ; 0, \hat{\varphi})$ de $(4.20)$ é dado por

$$
\begin{aligned}
\lim _{t \rightarrow \infty} y(t) e^{\left(\frac{e^{-A}}{\mu_{0}} \int_{0}^{t} b(\theta) d \theta\right)} & \\
& =\frac{1}{\mu_{0}\left(\mu_{0}+D\right)}\left(\hat{\varphi}(0)+\frac{e^{-A}}{\mu_{0}} \int_{-1}^{0} b(s) e^{\left(\frac{e^{-A}}{\mu_{0}} \int_{s}^{0} b(\theta) d \theta\right)} \hat{\varphi}(s) d s\right) .
\end{aligned}
$$

Finalmente, voltemos à equação original, usando (4.19). Assim o comportamento assintótico da solução $x\left(\cdot ; t_{0} ; \varphi\right)$ da equação $(4.18)$ é dado por

$$
\begin{aligned}
& \lim _{t \rightarrow \infty} x(t) e^{\left(\frac{e^{-A}}{\mu_{0}} \int_{t_{0}}^{t} b\left(\tau-t_{0}\right) d \tau-\int_{t_{0}}^{t}\left(\tau-t_{0}\right) d \tau\right)} \\
= & \frac{1}{\mu_{0}\left(\mu_{0}+D\right)}\left(\varphi(0)+\frac{e^{-A}}{\mu_{0}} \int_{-1}^{0} b(s) e^{\left(\frac{e^{-A}}{\mu_{0}} \int_{s}^{0} b(\tau) d \tau-\int_{s}^{0} a(\tau) d \tau\right)} \varphi(s) d s\right) .
\end{aligned}
$$




\section{ApÊNDice A \\ Dominância de Raízes de Equações Características}

No estudo do comportamento assintótico das soluções de uma EDF é importante determinarmos se um certo zero da equação característica é dominante. A função

$$
\Delta(z)=z\left[1-\int_{0}^{r} e^{-z \theta} d \mu(\theta)\right]-\int_{0}^{r} e^{-z \theta} d \eta(\theta)
$$

aparece tipicamente como equação característica no estudo das EDF. Restringindo as funções $\mu$ e $\eta$ a funções escada, podemos escrever (A.1) na forma

$$
\Delta(z)=z\left(1+\sum_{l=1}^{n} c_{l} e^{-z \sigma_{l}}\right)+a+\sum_{j=1}^{m} b_{j} e^{-z \tau_{j}} .
$$

Neste trabalho por exemplo, na Seção (4.1), estudamos a dominância das raízes da equação

$$
2 \lambda\left(e^{\lambda}-1\right)-1=0,
$$

o que nos custou para mostrar a dominância de um certo zero. Esta equação equivale à equação

$$
\lambda\left(1-e^{-\lambda}\right)-\frac{e^{-\lambda}}{2}=0 .
$$

A função no lado esquerdo de (A.4) pode ser escrita na forma (A.2), tomando $n=m=1$, os retardamentos $\sigma_{1}=\tau_{1}=1 \mathrm{e}$ os coeficientes $c_{1}=-1, a=0 \mathrm{e}$ $b_{1}=-1 / 2$.

Em Frasson [4], foi dada uma condição suficiente para que um determinado zero das funções (A.1) e (A.2) seja simples e dominante. Veja também 
Frasson \& Verduyn Lunel [3]. Incluímos também um resultado sobre uma função típica em equações características de equações simples, que apareceu primeiro em Frasson \& Verduyn Lunel [3]. Daremos estes resultados aqui, com suas provas. Estes resultados, em conjunto com os resultados do capítulo 3 fornecem extensões aos resultados em Philos \& Purnaras [8], sendo resultados inéditos.

\section{A.1 Uma condição para a dominância de raízes}

Para estudarmos (A.1) definimos uma função auxiliar $V: \mathbb{C} \rightarrow[0, \infty)$ por

$$
V(z)=\int_{0}^{r}(1+|z| \theta)\left|e^{-z \theta}\right| d|\mu|(\theta)+\int_{0}^{r} \theta\left|e^{-z \theta}\right| d|\eta|(\theta),
$$

onde $|\mu|$ e $|\eta|$ denotam respectivamente as funções variação total $\mu$ e $\eta$ na equação (A.1).

Teorema A.1 Suponha que $z_{0} \in \mathbb{C}$ seja um zero de $\Delta(\cdot)$ em (A.1) tal que

$$
V\left(z_{0}\right)<1
$$

Então $z_{0}$ é um zero simples e dominante de $\Delta(\cdot)$.

Demonstração: Para mostrarmos que $z_{0}$ é um zero simples de $\Delta(\cdot)$, calculemos

$$
\frac{d}{d z} \Delta(z)=1+\int_{0}^{r} e^{-z \theta}(-1+z \theta) d \mu(\theta)+\int_{0}^{r} \theta e^{-z \theta} d \eta(\theta) .
$$

Então podemos estimar

$$
\begin{aligned}
\left|\frac{d}{d z} \Delta\left(z_{0}\right)\right| & \geqslant 1-\left|\int_{0}^{r} e^{-z_{0} \theta}\left(-1+z_{0} \theta\right) d \mu(\theta)+\int_{0}^{r} \theta e^{-z_{0} \theta} d \eta(\theta)\right| \\
& \geqslant 1-V\left(z_{0}\right)>0,
\end{aligned}
$$

Que mostra que $z_{0}$ é um zero simples de $\Delta(\cdot)$. Resta-nos provar que $z_{0}$ é dominante. Para isso, seja $0<\delta<1$ tal que

$$
V\left(z_{0}\right)<\delta
$$


Podemos reescrever (A.7) para obter

$$
\begin{aligned}
1-\frac{1}{\delta} \int_{0}^{r}\left|e^{-z_{0} \theta}\right| & d|\mu|(\theta) \\
> & \frac{1}{\delta}\left[\left|z_{0}\right| \int_{0}^{r} \theta\left|e^{-z_{0} \theta}\right| d|\mu|(\theta)+\int_{0}^{r} \theta\left|e^{-z_{0} \theta}\right| d|\eta|(\theta)\right] .
\end{aligned}
$$

Seja $\epsilon>0$ tal que

$$
1<e^{\epsilon r} \leqslant \frac{1}{\delta},
$$

e $\Lambda$ o semiplano direito dado por

$$
\Lambda=\left\{z \in \mathbb{C}: \operatorname{Re} z>\operatorname{Re} z_{0}-\epsilon\right\} .
$$

Para $z \in \Lambda$ e $0 \leqslant \theta \leqslant r$ temos que

$$
\left|e^{-z \theta}\right| \leqslant e^{-\operatorname{Re} z_{0} \theta} e^{\epsilon \theta} \leqslant \frac{\left|e^{z_{0} \theta}\right|}{\delta} .
$$

Seja $z \in \Lambda$ e $\gamma$ o seguimento de reta que liga $z_{0}$ a $z$, que está contido em $\Lambda$. Portanto, para $0 \leqslant \theta \leqslant r$, de (A.11),

$$
\left|e^{-z_{0} \theta}-e^{-z \theta}\right|=\left|\int_{z_{0}}^{z} \theta e^{-w \theta} d w\right|=\theta\left|\int_{\gamma} e^{-w \theta} d w\right| \leqslant \frac{\left|e^{z_{0} \theta}\right|}{\delta}\left|z-z_{0}\right| \theta .
$$

Como $\Delta\left(z_{0}\right)=0$, obtemos que

$$
z_{0}=z_{0} \int_{0}^{r} e^{-z_{0} \theta} d \mu(\theta)+\int_{0}^{r} e^{-z_{0} \theta} d \eta(\theta),
$$

que podemos usar para reescrever $\Delta(z)$ como segue.

$$
\begin{gathered}
\Delta(z)=\left(z-z_{0}\right)\left[1-\int_{0}^{r} e^{-z \theta} d \mu(\theta)\right]+z_{0}-z_{0} \int_{0}^{r} e^{-z \theta} d \mu(\theta)-\int_{0}^{r} e^{-z \theta} d \eta(\theta) \\
=\left(z-z_{0}\right)\left[1-\int_{0}^{r} e^{-z \theta} d \mu(\theta)\right] \\
\quad+z_{0} \int_{0}^{r}\left(e^{-z_{0} \theta}-e^{-z \theta}\right) d \mu(\theta)+\int_{0}^{r}\left(e^{-z_{0} \theta}-e^{-z \theta}\right) d \eta(\theta) .
\end{gathered}
$$


Assim podemos estimar

$$
\begin{aligned}
|\Delta(z)| \geqslant \mid z & -z_{0}|| 1-\int_{0}^{r} e^{-z \theta} d \mu(\theta) \mid \\
& -\left|z_{0} \int_{0}^{r}\left(e^{-z_{0} \theta}-e^{-z \theta}\right) d \mu(\theta)+\int_{0}^{r}\left(e^{-z_{0} \theta}-e^{-z \theta}\right) d \eta(\theta)\right| .
\end{aligned}
$$

Para $z \in \Lambda$, de (A.11), chegamos a

$$
\left|1-\int_{0}^{r} e^{-z \theta} d \mu(\theta)\right| \geqslant 1-\left|\int_{0}^{r} e^{-z \theta} d \mu(\theta)\right| \geqslant 1-\frac{1}{\delta} \int_{0}^{r}\left|e^{-z_{0} \theta}\right| d|\mu|(\theta) .
$$

Da (A.8) e (A.15), obtemos

$$
\left|1-\int_{0}^{r} e^{-z \theta} d \mu(\theta)\right|>\frac{1}{\delta}\left[\left|z_{0}\right| \int_{0}^{r} \theta\left|e^{-z_{0} \theta}\right| d|\mu|(\theta)+\int_{0}^{r} \theta\left|e^{-z_{0} \theta}\right| d|\eta|(\theta)\right] .
$$

Usando (A.12), estimamos

$$
\begin{aligned}
& \left|z_{0} \int_{0}^{r}\left(e^{-z_{0} \theta}-e^{-z \theta}\right) d \mu(\theta)+\int_{0}^{r}\left(e^{-z_{0} \theta}-e^{-z \theta}\right) d \eta(\theta)\right| \\
& \quad \leqslant \frac{\left|z-z_{0}\right|}{\delta}\left[\left|z_{0}\right| \int_{0}^{r} \theta\left|e^{-z_{0} \theta}\right| d|\mu|(\theta)+\int_{0}^{r} \theta\left|e^{-z_{0} \theta}\right| d|\eta|(\theta)\right] .
\end{aligned}
$$

Finalmente, se $z \in \Lambda$ e $\left|z-z_{0}\right|>0$, de (A.14), (A.16) e (A.17) temos

$$
|\Delta(z)|>0 \text {. }
$$

Portanto o único zero de $\Delta(\cdot)$ que está dentro do semiplano direito $\Lambda$ é $z_{0}$. Isto completa a demonstração.

No caso da função característica ser da forma (A.2), o teorema A.1 toma a forma do corolário a seguir, que apareceu inicialmente em [3, Lema B.1].

Corolário A.2 Se $z_{0}$ é um zero de (A.2) tal que

$$
\sum_{l=1}^{n}\left|c_{l}\right|\left(1+\left|z_{0}\right| \sigma_{l}\right)\left|e^{-z_{0} \sigma_{l}}\right|+\sum_{j=1}^{m}\left|b_{j}\right| \tau_{j}\left|e^{-z_{0} \tau_{j}}\right|<1,
$$

então $z_{0}$ é um zero simples e dominante (A.2). 
Demonstração: A equação característica $\Delta(\cdot)$ dada em (A.2) pode ser colocada na forma (A.1) se colocamos as funções $\eta$ e $\mu$ como funções escada respectivamente com "saltos" de tamanho $c_{l}$ em $\sigma_{l}$ e $b_{j}$ em $\tau_{j}$. Portanto as funções variação total $|\eta|$ e $|\mu|$ são igualmente funções escada com saltos de tamanho $\left|c_{l}\right|$ em $\sigma_{l}$ e $\left|b_{j}\right|$ em $\tau_{j}$ respectivamente. Então a condição $V\left(z_{0}\right)<1$ no Teorema A.1 pode ser escrita como (A.19) e segue o resultado.

\section{A.2 Uma equação simples}

Agora restringimos nossa atenção à função

$$
\lambda-a-b e^{-\tau \lambda}=0,
$$

onde $a, b \in \mathbb{R}$ e $b \neq 0$. Esta função apareceu como equação característica de EDF estudadas por Driver [2] e Kordonis, Niyianni \& Philos [7].

Para $x \in[-1, \infty)$ nos temos que $x \mapsto x e^{x}$ é uma função estritamente crescente com imagem em $\left[-e^{-1}, \infty\right)$. Nós denotamos sua inversa como $W(\cdot)^{1}$. Simbolicamente, temos

$$
y=x e^{x}, x \geqslant-1 \Longleftrightarrow x=W(y) .
$$

Para $-e^{-1}<y<0$, a equação

$$
y=x e^{x}
$$

tem duas soluções reais. Observamos que $W(y)$ é a maior delas. Se $y<-e^{-1}$ então (A.22) não admite solução real $x$.

O teorema a seguir foi tirado de Frasson [4], e é um pequeno melhoramento do Lema B.3 em Frasson \& Verduyn Lunel [3].

Teorema A.3 $S e-e^{-1}<b \tau e^{-a \tau}$, então a equação

$$
\Delta(z)=z-a-b e^{-\tau z}
$$

tem um zero real, simples e dominante $z=\lambda_{d}$ dado por

$$
\lambda_{d}=a+\frac{1}{\tau} W\left(b \tau e^{-a \tau}\right),
$$

onde a função $W$ é dada por (A.21).

\footnotetext{
${ }^{1}$ A função $W(\cdot)$ é conhecida como a função $W$ de Lambert. Nos sistemas de computação algébrica Maple e Mathematica, podemos acessar esta função sob os nomes LambertW e ProductLog respectivamente.
} 
Demonstração: Podemos reescrever $\Delta(z)=0$ na forma equivalente

$$
(z-a) \tau e^{(z-a) \tau}=b \tau e^{-a \tau} .
$$

Então a representação (A.24) para $\lambda_{d}$ e a não existência de raízes reais de (A.23) quando $-e^{-1}>b \tau e^{-a \tau}$ segue da discussão sobre a função $W$. Diferenciando $\Delta(z)$ com respeito a $z$, calculando seu valor em $z=\lambda_{d}$ e usando as propriedades da função $W(\cdot)$, obtemos

$$
\frac{d}{d z} \Delta\left(\lambda_{d}\right)=1+W\left(b \tau e^{-a \tau}\right)
$$

Portanto $\frac{d}{d z} \Delta\left(\lambda_{d}\right)=0$ se, e só se, $-e^{-1}=b \tau e^{-a \tau}$. Temos $\left|\frac{d^{2}}{d z^{2}} \Delta(z)\right|>0$ para todo $z \in \mathbb{C}$. Assim, com a hipótese do teorema, $\lambda_{d}$ é um zero simples de $\Delta(z)$. Notemos também que segue da representação (A.24) que, $b \mapsto \lambda_{d}$ é uma função estritamente crescente em $b$, e $\lambda_{d}=a$ for $b=0$. Resta-nos provar que $\lambda_{d}$ é dominante. Suponhamos primeiro que $-e^{-1}<b \tau e^{-a \tau}<0$ (portanto $b<0$ ). Recordando a equação (A.5), temos

$$
V\left(\lambda_{d}\right)=|b| \tau e^{-\lambda_{d} \tau}=\left|W\left(b \tau e^{-a \tau}\right)\right| .
$$

Quando $-e^{-1}<b \tau e^{-a \tau}<0$ temos que $V\left(\lambda_{d}\right)<1$, assim as hipóteses do Corolário A.2 estão satisfeitas e segue que $\lambda_{d}$ é um zero simples e dominante de $\Delta(z)$. Consideramos agora o caso $b>0$. Então $\lambda_{d}>a$. Suponha que $z=x+i y$ é outro zero de $\Delta$ com $x, y$ números reais e $y>0$. As equações para as partes real e imaginária para $z$ são dadas por

$$
\begin{gathered}
x-a-b \cos (\tau y) e^{-\tau x}=0, \\
y+b e^{-\tau x} \sin (\tau y)=0 .
\end{gathered}
$$

Definimos $\bar{b}=b \cos (\tau y)$. A equação (A.27) torna-se

$$
x-a-\bar{b} e^{-\tau x}=0 .
$$

Se $\bar{b} \leqslant 0$, então (A.29) e os argumentos já dados implicam que $x \leqslant a<\lambda_{d}$. Se $\bar{b}>0$ então (A.28) e $y>0$ implicam que $\tau y \neq k \pi$ para todo inteiro $k$ e portanto $\bar{b}<b$, mas isto de novo implica que $x<\lambda_{d}$. Para termos a dominância, devemos mostrar que existe um $\epsilon>0$ tal que, se $z$ é outro zero de (A.23), então $\operatorname{Re} z<\operatorname{Re} \lambda_{d}-\epsilon$. Suponhamos por absurdo que tal $\epsilon$ não existe. Assim existe uma sequência $z_{n}$ de zeros de $\Delta(\cdot)$ tais que $\operatorname{Re} z_{n} \rightarrow \lambda_{d}$. 
Como $\Delta(\cdot)$ é uma função analítica, seus zeros são isolados, e portanto a única possibilidade é que $\left|\operatorname{Im} z_{n}\right| \rightarrow \infty$, mas (A.28) mostra-nos que $\operatorname{Im} z_{n}$ é um conjunto limitado, o que é absurdo. Portanto, também no caso $b>0, \lambda_{d}$ é um zero simples e dominante de $\Delta(\cdot)$. 


\section{Referências Bibliográficas}

[1] Richard Bellman and Kenneth L. Cooke. Differential-difference equations. Academic Press, New York, 1963.

[2] R. D. Driver, D. W. Sasser, and M. L. Slater. The equation $x^{\prime}(t)=$ $a x(t)+b x(t-\tau)$ with "small" delay. Amer. Math. Monthly, 80:990-995, 1973.

[3] Miguel V. S. Frasson and Sjoerd M. Verduyn Lunel. Large time behaviour of linear functional differential equations. Integral Equations Operator Theory, 47(1):91-121, 2003.

[4] M.V.S. Frasson. On the dominance of roots of characteristic equations for neutral functional differential equations. Preprint, 2008.

[5] Israel Gohberg, Seymour Goldberg, and Marinus A. Kaashoek. Classes of linear operators. Vol. I, volume 49 of Operator Theory: Advances and Applications. Birkhäuser Verlag, Basel, 1990.

[6] Jack K. Hale and Sjoerd M. Verduyn Lunel. Introduction to functionaldifferential equations, volume 99 of Applied Mathematical Sciences. Springer-Verlag, New York, 1993.

[7] I.-G. E. Kordonis, N. T. Niyianni, and c. G. Philos. On the behavior of the solutions of scalar first order linear autonomous neutral delay differential equations. Arch. Math. (Basel), 71(6):454-464, 1998.

[8] Ch. G. Philos and I. K. Purnaras. Periodic first order linear neutral delay differential equations. Appl. Math. Comput., 117(2-3):203-222, 2001.

[9] H. L. Royden. Real analysis. Macmillan Publishing Company, New York, third edition, 1988. 
[10] Walter Rudin. Real and complex analysis. McGraw-Hill Book Co., New York, third edition, 1987.

[11] Angus Ellis Taylor and David C. Lay. Introduction to functional analysis. John Wiley \& Sons, New York-Chichester-Brisbane, second edition, 1980 . 


\section{Índice Remissivo}

Atomicidade em zero, 14

uniformemente atômico, 15

uniformemente não atômico, 18

Autovalor

de tipo finito, 28

dominante, 27

simples, 22

$\mathcal{C}$, espaço das funções contínuas, 14 $C_{z}, 35$

$e^{a_{D} \omega}$, raio espectral de $T_{D}(\sigma+\omega, \sigma)$ 28

EDFN

autônoma, 15

linear, 15

homogênea, 15

não-homogênea, 15

EDFR, 15

$E_{\mu}, 22$

Expoente

característico, 22,27

Função

$W$ de Lambert, 45,55

$G_{\varphi, z}, 35$

$\mathcal{L}$, operadores lineares contínuos, 21 $L_{1}^{\text {loc }}$, funções localmente integráveis, 17
Multiplicador

característico, 22, 27

de EDFN, 28

simples, 38

de Floquet, 22

Multiplicidade, 22

$\mathcal{N}$, núcleo de operador, 28

NBV, funções de variação limitada normalizadas, 14

$\Omega_{s}^{t}\left(\frac{1}{z}\right), 35,36$

Operador

diferença, 15

monodrômico, 22

solução, 19

П, operador monodrômico, 22

$P_{\mu}$, projeção espectral, 25,38

P $\sigma(\Pi)$, espectro pontual de $\Pi, 22$

$Q_{\mu}, 22$

$\mathcal{R}$, imagem de operador, 28

Representação de Floquet, 25

$\sigma(\Pi)$, espectro de $\Pi, 22,38$

Solução

continuação, 17

de tipo Floquet, 24

não-continuável, 17

$T(t, s)$, operador solução, 19 
$T_{D}(t), 19,28$

$T_{D, f}(t), 19,28$

$W, 45,55$

$x(\cdot ; \sigma, \varphi), 15$

$x_{t}, 14$ 